\title{
Die Ranunculaceae der Flora von Zentraleuropa: Ficaria $^{1}$
}

WALTER GUTERMANN, Division of Systematic and Evolutionary Botany, University of Vienna, Rennweg 14, Vienna, 1030, Austria, e-mail: walter.gutermann@univie.ac.at

\section{Ficaria $^{2}$}

Guettard 1754, Hist. Acad. Roy. Sci. Mém. Math. Phys. (Paris, $4^{\circ}$ ) 1750: 355. - Syn.: Ranunculus sect. Ficaria (GUETT.) BENTH. in BENTHAM \& HOOKER 1862, Gen. Pl. 1: 6; Ranunculus subgen. Ficaria (GUETT.) L.D. BENSON 1940, Amer. J. Bot. 27: 807. - S c h a r b o c k s k r a u t, Feigwurz. Engl.: lesser celandine; dän.: vorterod; niederl.: speenkruid; franz.: ficaire; ital.: ficaria, favagello; slowen.: lopatica; ungar.: salátaboglárka; slowak.: blyskáč; tschech.: orsej; sorb.: bórliški; poln.: ziarnopłon; litau.: švitriešis; russ.: чистяк [čistjak].

Typus-Art: Ficaria verna HUDS. [Ranunculus ficaria L.]

Literatur [Weitere, ältere Arbeiten sind bei WOLTER (1933) angeführt]. ANDREAS, Ch.H. 1954: Notes on Ranunculus ficaria L. in the Netherlands. I. Introduction. Reductional trends as a possible interpretation of flower types. Acta Bot. Neerl. 3: 446-453; 1966: Enkele infraspecifieke taxa bij Ranunculus ficaria L. Gorteria 3: 11-12. - ANDERS-GASSER, I. 1985: Cytotaxonomische Untersuchungen an Ranunculus ficaria L. Mitt. Naturf. Ges. Bern, n. s., 42: 79-85. - AUGSTEN, H. 1957: Die Wirkung tiefer Temperaturen auf die Entwicklung von Ranunculus ficaria L. Ber. Deutsch. Bot. Ges. 70: 233-244; 1967: Zur Beziehung zwischen Nachreife und Zuckerstoffwechsel bei Bulbillen von Ficaria verna HudS. Flora 158: 604-608. - AvANCINI, F., MATTEUCCI, M. \& LuCCHESE, F. 2007: Identificazione delle popolazione del gruppo Ranunculus ficaria L. dei Monti della Tolfa (Lazio). Inform. Bot. Ital. 39 (Suppl. 1): 172-178. - AXTELL, A. E., DiTOMMASO, A. \& POST, A. R. 2010: Lesser celandine (Ranunculus ficaria): A threat to woodland habitats in the Northern United States and Southern Canada. Invasive Pl. Sci. Managem. 3: 190-196. - BALTISBERGER, M. \& HöRANDL, E. 2016: Karyotype evolution supports the molecular phylogeny in the genus Ranunculus (Ranunculaceae). Perspect. Pl. Ecol. Evol. Syst. 18: 1-14. - BARros NeVES, J. 1942: Sôbre a cariologia de Ranunculus ficaria L. Bol. Soc. Brot., ser. 2, 16: 169-181. - BENSON, L. 1940: The North American subdivisions of Ranunculus. Amer. J. Bot. 27: 799-807; 1954: Supplement to a treatise on the North American Ranunculi. Amer. Midl. Naturalist 52: 328-369. - BuGNON, P. 1933: Sur l'origine de la monocotylie chez la ficaire. Compt. Rend. Hebd. Séances Acad. Sci. LIXVI: 1918. - CouRdUROUX, J.-C. 1966: Tubérisation et biologie de la ficaire (Ficaria ranunculoides MoENCH). Physiol. Vég. 4: 341-364. - DOISDADO, J.C. \& PASTOR, J. 1993:

\footnotetext{
1 Zitierempfehlung: GutermanN, W. 2021: Die Ranunculaceae der Flora von Zentraleuropa: Ficaria. - Publiziert unter http://www.flora-deutschlands.de/ranunculaceae.html

2 lat. ficarius, Adjektiv zu lat. ficus »Feige«, in substantivischer Form bereits als vorlinnäischer Gattungsname (so etwa bei DILLENIUS); nach der Form der Speicherwurzeln.
} 
Aportación al conocimiento cariológico del género Ranunculus L. subgénero Ficaria (SCHAEFFER) L. BENSON en la Península Ibérica. Acta Bot. Malacitana 18: 77-88. - DRENCKHAHN, D. 2016: Morphologie und Jahreszyklus von Ficaria calthifolia RCHB. - eine neu etablierte Sippe in Deutschland. Forum Geobot. 7: 1-17. - DrencKhahn, D., BAUMGARTNER, W. \& ZONNEVELD, B. 2017: Different genome sizes of Western and Eastern Ficaria verna lineages shed light on steps of Ficaria evolution. Forum Geobot. 7: 27-33 - DosTÁL, L. 1927: [in: WINKLER, U.: Bausteine zu einer Monographie von Ficaria.] 6. Über die Rhythmikabhängigkeit der morphogenen Beziehungen zwischen Speicher- und Absorptionswurzeln bei Ficaria verna. Beitr. Biol. Pflanzen 15: 376-416, t. VIII. - EMADZADE, K., LEHNEBACH, C., LOCKHART, P. \& HÖRANDL, E. 2010: A molecular phylogeny, morphology and classification of genera of Ranunculeae (Ranunculaceae). Taxon 59: 809-828. - FÖRSTER, P. 1997: Die Keimpflanzen der Tribus Ranunculeae DC. und der Tribus Adonideae KUNTH (Ranunculaceae). Flora 192: 133-142. - FRANK, D. 2017: Man sieht nur, was man kennt. Ficaria calthifolia RCHB. auf historisch altem Grünland. Mitt. florist. Kart. Sachsen-Anhalt 22: 23-32. - FUTÁK, J. 1982: Ficaria SCHAEFFER. In: FUTÁK, J. \& BerTOvÁ, L. (Eds.): Flóra Slovenska 3: 215-218. VEDA, Bratislava. - GAMS, H. 1932: [in: WINKLER, U.: Bausteine zu einer Monographie von Ficaria.] 7. Ranunculus ficaria als amphibische Hydrochore. Beitr. Biol. Pflanzen 19: 228-231. - GILL, J.J.B., JoNES, B.M.G., MARChANT, C.J., MCLEISH, J. \& OCKENDON, D.J. 1972: The distribution of chromosome races of Ranunculus ficaria L. in the British Isles. Ann. Bot. (London), n. s., 36: 31-47. - GOEPPERT, R. 1831: Ueber die sogenannten Getreide- und Schwefel-Regen. I. Ueber Getreide-Regen. Ann. Physik 97(4): 550-572. - GREEN, P.S. \& THOMAS, J.L. 1961: The bulbiferous Ranunculus ficaria. Rhodora 63: 289-291. - GREILHUBER, J. 1974: Ein Chromosomensatz von Ranunculus ficaria subsp. calthifolius. Mitt. Bot. Arbeitsgem. Oberösterr. Landesmus. 6: 1-3. - HARPER, P.J. 1999: The greater pleasures of lesser celandines. Horticulture 96(3): 32-34. - Hörandl, E., PAun, O., Johansson, J.T., Lehnebach, C., Armstrong, T., Chen, L. \& LOCKHART, P. 2005: Phylogenetic relationships and evolutionary traits in Ranunculus s. 1. (Ranunculaceae) inferred from ITS sequence analysis. Molec. Phylogen. Evol. 36: 305-327. - ILLIG, H. \& Ristow, M. 2015: Ficaria calthifolia RCHB. (syn. Ranunculus ficaria subsp. calthifolius [RCHB.] ARCANG.) - über einen Neufund für Deutschland. Verh. Bot. Vereins Berlin Brandenburg 147: 13-49. - IRMISCH, TH. 1854: Beiträge zur vergleichenden Morphologie der Pflanzen 1: Ranunculus ficaria L. Abh. Naturf. Ges. Halle 2: 31-80. - JOHANSSON, J.T. 1998: Chloroplast DNA restriction site mapping and the phylogeny of Ranunculus (Ranunculaceae). Pl. Syst. Evol. 213: 1-19. - JONES, B.M.G. 1975: Subg. Ficaria (Huds.) L. BenSON. In: STACE, C.A. (Ed.): Hybridization and the flora of the British Isles: 129-130. - KÄSTNER, A. \& FISCHER, M.A. 2006: Porträts ausgewählter seltener österreichischer Gefäßpflanzenarten (II). Neilreichia 4: 75-109. - KERMACK, J.P. \& RAUSCHERT, E.S.J. 2019: Soil characteristics drive Ficaria verna abundance and reproductive output. Invasive Pl. Sci. Managem. 12: 214 222. - KINDLER, T. 1914: Gametophyt und Fruchtansatz bei Ficaria ranunculoides. Österr. Bot. Z. 64: 73-85. - KLOOSS, S., FISCHER, E., OUT, W. \& KIRLEIS, W. 2015: Charred root tubers of lesser celandine (Ficaria verna HUDS.) in plant macro remain assemblages from Northern, Central and Western Europe. Quatern. Int. 404: 25-42. - KRIŽO, M. \& KORÍNEKOVÁ, M. 1987: The life cycle of Ficaria verna HuDS. Preslia 59: 243-251. - KÜNZEL, R. 1980: Ranunculus ficaria L. (Ranunculaceae) in Berlin: zur Karyologie und Morphologie einer kritischen Sippe. Verh. Bot. Vereins Prov. Brandenburg 115: 73-108. LAEGAARD, S. 1966: Ranunculus ficaria ssp. fertilis in Denmark. Bot. Tidsskr. 61: 295-297; 2001: Validation of Ranunculus ficaria L. ssp. fertilis CLAPHAM ex LAEGAARD (Ranunculaceae). Nordic J. Bot. 20: 525-526. - LAMBINON, J. 1981: Le nom correct de la ficaire à bulbilles: Ranunculus ficaria L. subsp. bulbilifer LAMBINON nom. nov. Bull. Jard. Bot. Natl. Belg. 51: 461-463. - LOSCHNIGG, F. 1925: [in: WINKLER, U.: Bausteine zu einer Monographie von Ficaria.] 4. Über die Ursachen der häufigen Sterilität. Beitr. Biol. Pflanzen 14: 347-358, t. VII. - MARCHAND, C.J. \& BRIGHTON, C.A. 1975: Cytological diversity and triploid frequency in a complex population of Ranunculus ficaria L. Ann. Bot. 
(London), n. s., 38: 7-15. - MARSDEN-JONES, E.M. 1935: Ranunculus ficaria L.: life history and pollination. J. Linn. Soc., Bot. 50: 39-55; 1937: Pollination of Ranunculus ficaria L. by insects. J. Bot. 75: 133-141. - Marsdon, A., Cabo, M., Lubrano, C., Robin, R.-J., Fromeageot, C. \& Hostettmann, K. 2006: Classification of the saponin composition of Ranunculus ficaria tubers. Nat. Prod. Commun. 1: 22-32. - MATTERN, A. 1995. Attraktive Sorten des Scharbockskrauts. Gartenpraxis 21(3): 8-13. METCALFE, C.R. 1936: An interpretation of the morphology of the single cotyledon of Ranunculus Ficaria, based on embryology and seedling anatomy. Ann. Bot. (Oxford) 50: 103-120; 1938: The morphology and mode of development of the axillary tubercules and root tubers of Ranunculus Ficaria. Ann. Bot. (Oxford), n. s., 2: 145-157; 1939: The sexual reproduction of Ranunculus Ficaria. Ann. Bot. (Oxford), n. s., 3: 91-103. - MUDRACK, F. 1935: Über die Assimilationstätigkeit und das Wachstum von Ficaria verna. Planta 23: 71-104. - NEGODI, G. 1930: Considerazioni sull'assetto cromosomico del Ranunculus ficaria L. e la sua frequente sterilità. Atti Soc. Naturalisti Modena 9: 167-172. - NeSOM, G.L. 2008: Ranunculus ficaria (Ranunculaceae), naturalized in Texas. J. Bot. Res. Inst. Texas 2: 741742. - NiCHOLSON, G.G. 1981: The use of chloroplast numbers in guard cells as a means of distinguishing the chromosome races of Ranunculus ficaria L. Ann. Bot. (Oxford), n. s., 48: 909-913. - PASZKO, B., PAUl, W., NiKel, A. \& Musiat, L. 2020: Typifications, a new synonym and new distribution data in Ficaria (Ranunculaceae). Phytotaxa 432: 144-154. - PAUn, O., LEHNEBACH, C., JOHANSSEN, J.T., LOCKHART, P. \& HÖRANDL, E. 2005: Phylogenetic relationships and biogeography of Ranunculus and allied genera (Ranunculaceae) in the Mediterranean region and in the European Alpine system. Taxon 54: 911-930. - PERJE, A.-M. 1952: Some causes of variation in Ranunculus Ficaria L. Ark. Bot., n. s., 2: 251-264. - POGAN, E. \& WCISŁO, H. 1973: Studies in Ranunculus ficaria L. I. Karyological analysis of Ranunculus ficaria L. ssp. bulbifer (MARSDEN-JONES) LAWALRÉE and R. ficaria L. ssp. calthifolius (RCHB.) ARC. Acta Biol. Cracov., ser. Bot., 16: 135-143, t. 18; 1974: II. Further karyological studies. Acta Biol. Cracov., ser. Bot., 17: 165-173; 1975: III. Karyotype analysis. Acta Biol. Cracov., ser. Bot., 18: 79-99, t. 21-22; 1981a: IV. Cyto-embryological studies. Acta Biol. Cracov., ser. Bot., 23: 37-54, t. 7-9; 1981b: V. Cytoembryological analysis of Ranunculus ficaria L. subsp. calthifolius (RCHB.) ARC. with B-chromosomes. Acta Biol. Cracov., ser. Bot., 23: 83-96, t. 13; 1983: VI. Cytoembryological analysis of triploids from Poland. Acta Biol. Cracov., ser. Bot., 25: 43-55, t. 3; 1986: VII. Additions to chromosome numbers. Acta Biol. Cracov., ser. Bot., 28: 87-92. - PONERT, J. 1975: Ficaria grandiflora ROBERT - novyj vid dlja flory SSSR [Ficaria grandiflora ROBERT - a new species for the flora of the U. S. S. R.]. Bot. Žurn. [Bot. Zhurn. (Moskow \& Leningrad)] 60: 704-706. - POPELKA, O., TRÁVNíčEK, B., ŠIKOVÁ, P., JANDOVÁ, M. \& DUCHOSLAV, M. 2019: Natural hybridization between diploid Ficaria calthifolia and tetraploid Ficaria verna subsp. verna in central Europe: evidence from morphology, ecology and life-history traits. Preslia 91: 179-212. - POST, A. R, KRINGS, A., WALL, W. A. \& NEAL, J. C. 2009: Introduced Lesser Celandine (Ranunculus ficaria, Ranunculaceae) and its putative subspecies in the United States: a morphometric analysis. J. Bot. Res. Inst. Texas. 3: 193-209. - REISCH, Ch., SCHEITLER, S. 2008: Disturbance by mowing affects clonal diversity: the genetic structure of Ranunculus ficaria (Ranunculuaceae) in meadows and forests. Pl. Ecol. 201: 699-707. - ROLAND-HEYDACKER, F. 1964: Morphologie du pollen de Ficaria ranunculoides MOENCH. Ranunculaceae. Pollen \& Spores 6: 373-378. - Roland, F. 1968: Différentiation du sporoderme chez Ficaria ranunculoides MoENCH. Observation et evolution de „corps d'Ubisch“. Pollen \& Spores 9: 415-425. - SELL, P.D. 1991: A new subspecies of Ranunculus ficaria L. [in M.E. NEWTON (Ed.): Flora Europaea: Notulae systematicae ad Floram Europaeam spectantes. Series 2. No.4] Bot. J. Linn. Soc. 106: 117-118; 1994: Ranunculus ficaria L. sensu lato. Watsonia 20: 41-50. - ŠIKOVÁ, P. 2014: Mezidruhová hybridizace v rodu Ficaria v prostoru střední Evropy. Interspecific hybridisation in the genus Ficaria at the area of Central Europe [p. 5: English abstract]. Diplomová práce, Univerzita Palackého v Olomouci, Olomouc. 57 pp., app. 1-4. - SOÓ, R. \& BORHIDI, A. 1966: Über einige Formenkreise der ungarischen und karpatischen Flora. 
IV. Ficaria. Ann. Univ. Sci. Budapest 8: 297-300. - TAMURA, M. 1995: Angiospermae: Ordnung Ranunculales. Fam. Ranunculaceae. Systematic part. In: ENGLER, A. \& PRANTL, K. (Begr.): Die Natürlichen Pflanzenfamilien, 2. Aufl. (Herausg.: P. HIEPKO), 17aIV: 223-497. Berlin: Duncker \& Humblot. - TAYLOR, K. \& MARKHAM, B. 1978: Ranunculus ficaria L. (Ficaria verna HUDS.; F. ranunculoides MoENCH). J. Ecol. 66: 1011-1031 [= Biological flora of the British Isles (Ed.: P.J. GRUBB), No. 142]. - TOMCZYK, M. \& GUDEJ, J. 2003: Quantitative analysis of flavonoids in the flowers and leaves of Ficaria verna HUDS. Z. Naturf., C 58: 762-764. - TOWPASZ, K. 1971: Ficaria verna Huds. ssp. calthifolia (RCHB.) VEL. w Polsce. Ficaria verna HUDS. ssp. calthifolia (RCHB.) VEL. in Poland. Fragm. Florist Geobot. 17: 215-219. - TRÖHLER, A. 1976: Zytotaxonomische Untersuchungen an Ranunculus ficaria L. Mitt. Naturf. Ges. Bern, n. s., 33: 22-28. - TzVELEV, N.N. 2007: Ficaria GuETT. In: TzVELEV, N.N. (Ed.): Flora of Russia 10A: 211-213. London: Taylor \& Francis. - VÁSQUEZ PARDO, F.M. 2016: Aproximación al conocimiento del género Ficaria Huds., (Ranunculaceae) en Extremadura, España. Folia Bot. Estremadurensis 9: 15-23. - VELDKAMP, J.F. 2015: De nomenclatuur van Speenkruiden (Ficaria verna HUDS. s. 1., Ranunculaceae). Gorteria 37: 84-116. - VESELÁ, E. 1969: Beitrag zur Taxonomie der Art Ficaria verna HudS. Preslia 41: 313-322. - WCISŁO, H. \& PogAN, E. 1981: Cytoembryological aspects of reduced seed-setting in Ranunculus ficaria L. subsp. bulbifer (MARSDEN-JONES) LAWALRÉE. Acta Soc. Bot. Poloniae 50: 253-255. - WINKLER, U. 1926: Bausteine zu einer Monographie von Ficaria. [1. Über die Ruheperiode, besonders der Bulbillen; 2. Die Deutung der Keimpflanze; 3. Die Keimfrist]. Beitr. Biol. Pflanzen 14: 219-230; 335-347; 1927 [5. Weitere Beobachtungen an der Keimpflanze] Beitr. Biol. Pflanzen 15: 126-128. [Vgl. dazu auch LOSCHNIGG 1925, DosTÁL 1927, GAMS 1932, WOlTER 1933] - WOlTER, H. 1933: [in: WinKLER, U.: Bausteine zu einer Monographie von Ficaria.] 8. Über Bestäubung, Fruchtbildung und Keimung bei Ficaria verna. Beitr. Biol. Pflanzen 21: 219-255. - ZAJĄC, A. \& ZAJĄC, M. (Reds.) 2001: Atlas rozmieszcenia róslin naczyniowych w Polske (Distribution atlas of vascular plants in Poland). Inst. Bot. Univ. Jagielloński, Kraków. - ZONNEVELD, B.J.M. 2015: De verschillende genoomgewichten van Europese Ficaria HUDS. (Ranunculaceae) duiden op acht soorten / Genome sizes of Ficaria HUDS. (Ranunculaceae) indicate eight separate species. Gorteria 37:118-139.

Wurzelknollen-Staude. Wurzeln z. T. fadenförmig (verzweigte Nährwurzeln), z. T. keulenfömig verdickt (stoffspeichernde Wurzelknollen). Grundachse ein stark (scheibenförmig) verkürzter, \pm unterirdischer Achsenkörper. Jahressprosse (Laub- und Blühtriebe) gestaucht oder mit entwickelten, beblätterten, z. T. verzweigten Stängeln. Blätter teils spreitenlos (scheidenförmig verbreiterte, grundständige, häutige Niederblätter), teils \pm lang gestielte Laubblätter mit scheidenförmigem Grund. Grund- und stängelständige Laubblätter einander ähnlich, mit ungeteilter (höchstens lappig gekerbter), rundlicher bis breit eiförmiger, am Grund \pm herzförmiger, handnerviger Spreite, kahl, etwas fleischig, \pm glänzend, frühjahrsgrün, randlich mit punktförmigen Hydathoden (an den Nervenenden). Blüten gestielt, endständig, i. d. Regel zwittrig, mit kopfig gewölbtem, kahlem Blütenboden. Äußere Blütenhüllblätter (Kelchblätter) meist 3(-4) (ausnahmsweise auf 6 vermehrt), eiförmig, \pm konvex, am Grund schwach gesackt, häutig, weißlich bis gelblich, in der vorderen Hälfte grün, kahl, den Kronblättern locker anliegend, abfällig. Innere Blütenhüllblätter (nektarfertile Kronblätter) (6-) 8-11(-17), deutlich länger als die Kelchblätter, schmal eiförmig bis eiförmig, gelb (sehr selten weiß, orange), oberseits (mit Ausnahme des basalen Drittels) glänzend, am Grund mit einer kleinen klappen- bis seicht taschenförmigen Nektargrube, unterseits matt, gegen die Spitze zu grünlich. Staubblätter zahlreich. Fruchtblätter zahlreich, mit je 1 anatropen Samenanlage, Griffel sehr kurz bis fehlend; Sammelfrucht aus freien Nüsschen, diese verkehrt ei- bis birnförmig (seitlich stark gewölbt), mit konisch verlängerter Basis (Elaiosom) dem \pm kugeligen Fruchtboden aufsitzend, \pm ungeschnäbelt, reif einzeln abfallend, Oberfläche glatt, locker behaart oder kahl; Samen kugelförmig. 
Artenzahl und Verbreitung. Die artenarme Gattung ist in fast ganz Europa verbreitet; ihr Areal reicht von den Britischen Inseln und Süd-Skandinavien (an der norwegischen Küste vereinzelt bis $68^{\circ} \mathrm{n}$. Br.) bis zum Ural, West-Sibirien und NW-Kasachstan (und Alma-Ata, ob heimisch?), im Süden von den Kanarischen Inseln (Tenerife; ob heimisch?) und dem mediterranen Nordwestafrika über Sizilien, Kreta, Zypern, Kleinasien und Palästina bis in die Kaukasusländer, nach Persien und SW-Turkmenien. Außer den Sippen des Komplexes um $F$. verna (in Mitteleuropa noch $F$. ambigua und $F$. calthifolia; außerdem F. grandiflora: vgl. unten, im Anschluss an F. verna), die nahezu das gesamte Gattungsareal besetzen, sind nur zwei deutlicher getrennte Arten Vorderasiens bekannt: F. ficarioides (BORY \& CHAUB.) HALÁCSY [Ranunculus ficarioides BORY \& CHAUB.], vom Libanon, Transkaukasien und der südlichen Türkei westlich bis Griechenland (Peloponnes, südl. Pindos) reichend, und F. fascicularis K. KOCH [Ranunculus kochii LEDEB.] von Turkmenien über Transkaukasien, Iran und N-Irak bis in die Türkei. Eine der F. ficarioides ähnliche Sippe (,F. verna var. africana PAU“) kommt in Marokko vor.

Systematische Stellung und Gliederung. Schon sehr früh wurde die Verwandtschaft um Ficaria verna [Ranunculus ficaria] wegen einer Reihe morphologischer Besonderheiten (ein einzelnes, apikal gekerbtes Keimblatt, meist nur 3 äußere, aber vermehrte innere Blütenhüllblätter, Wurzelknollen) als Satellitengattung von der formenreichen Gattung Ranunculus abgetrennt, bei einer Vereinigung mit ihr aber zumindest als eigene Sektion (wie in der »Flora Europaea«: TUTIN \& al. 1963, auch noch 1993) oder als Untergattung (so BENSON 1940 und zuletzt TAMURA 1995) hervorgehoben. Jüngste, auf molekulargenetischen Analysen von Kern- und Chloroplasten-Gensequenzen beruhende, phylogenetische Untersuchungen (JOHANSSON 1998, HÖRANDL \& al. 2005, PAUN \& al. 2005, EMADZADE \& al. 2010) bekräftigen die Separierung als eigene, monophyletische Gattung, die am engsten mit Coptidium (mit ebenfalls 3-zähligem Kelch) als eher basaler Gattung der Ranunculeae und nicht unmittelbar mit $R a$ nunculus verwandt ist. Eine Vereinigung mit Ranunculus müsste nach diesen Befunden außer Coptidium auch Ceratocephala, Myosurus, Krapfia und Laccopetalum einschließen, die ihrerseits jeweils durch deutliche Apomorphien sowie karyologische Eigenheiten ausgezeichnet sind.

Die Ficaria-Sippen sind miteinander nahe verwandt, so dass sie manche Autoren zu einer einzigen Art zusammenfassen wollen. In Europa hebt sich $F$. ficarioides durch ringsum grob bis lappig gekerbte Laubblätter vom Komplex der F. verna ab, zu welchem außer den im Folgenden behandelten mitteleuropäischen Arten auch noch $F$. grandiflora ROBERT gehört, unter welchem Namen einstweilen die großblütigen mediterran(-atlantisch) verbreiteten, noch unzureichend bekannten Sippen zusammengefasst werden können (vgl. unten: im Anschluss an F. verna).

Morphologie. Die Keimung erfolgt epigäisch. Der Ficaria-Sämling besitzt ein bleibend kurzes Hypokotyl und nur ein Keimblatt mit relativ langem Stiel und einer eingekerbten oder zweilappigen, im embryonalen Zustand längs gefalteten Spreite. Manche ältere Autoren hielten das apikal 2- (manchmal 3-) lappige Organ für kongenital verwachsene Keimblätter; solche Keimpflanzen sind (allerdings nicht als Regelfall!) bei einzelnen Ranunculus-Arten nachgewiesen. (Bei dem wie Ficaria obligatorisch einkeimblättrigen $R$. glacialis ist die Keimblattspreite ungeteilt.) Gegen diese Deutung als „Synkotylie“ haben sich (nach IRMISCH 1854) vor allem WINKLER (1926) und GOEBEL (1932) ausgesprochen; dementsprechend sollte eines der Keimblätter im Laufe der Phylogenie verloren gegangen sein. Auch MARSDENJONES (1936) ist der Ansicht, dass es sich um ein einzelnes Blattorgan handelt, ebenso plädiert FÖRSTER (1997) in einer neueren Untersuchung für die „Monokotylie“ des Sämling bei Ficaria und deutet ihn als neotene Metamorphose im Zusammenhang mit der ephemeroiden Lebensweise.

Die homorrhizen Nährwurzeln werden am Grund der Erneuerungsknospen gebildet. Die keulig verdickten, unverzweigt bleibenden Speicherwurzeln sind (wie DOSTÁL 1927 auch experimentell gezeigt hat) 
Metamorphosen der gewöhnlichen Wurzeln, bei denen die Wurzelhaube verlorengeht; sie entspringen den Achselknospen der Niederblätter. Das Gefäßsystem wird in diesen Wurzelknollen rückgebildet, während ihre Rindenzellen Stärkekörner speichern. Der strenge Lebensrhythmus (autogene Sommerruhe) steuert diese, mit der sympodialen Sprossverkettung zusammenhängende, periodische und lokal begrenzte Wurzelbildung.

Der Sprossaufbau ist durch basitone Förderung gekennzeichnet; zum Hauptspross treten Seitensprosse, die den Achseln der basalen Blätter entspringen. Der nachträglichen Bildung der Achselsprosse geht die Aufblühfolge parallel: nach der Endblüte des Hauptsprosses folgen jene der oberen Seitensprosse, während die weiteren vegetativ bleiben. Die basale Achsenstauchung ist verantwortlich für den Scheinwirtel der Rosette, die sich gewöhnlich etwa im Niveau der Substratoberfläche entwickelt (und zwar über einem verkürzten oder entsprechend ausgebildeten Grundinternodium, je nach Lage der Grundachse). Bei $F$. verna, $F$. ambigua und $F$. grandiflora strecken sich oberhalb der Blattrosette mehrere Internodien der dann eher legtriebartigen Luftsprosse, so dass deren Laubblätter als Stängelblätter erscheinen. Die obersten Blätter des relativen Hauptsprosses und die transversalen Vorblätter der Seitensprosse stehen dabei nicht selten gegenständig, bezeugen aber durch unterschiedliche Größe ihre Stellung in einem Scheinwirtel.

Die bei den polyploiden Sippen von Ficaria in den Stängelblattachseln auftretenden Bulbillen sind Brutknospen, die den Erneuerungsknospen am Grund der Hauptachse entsprechen (wie IRMISCH schon 1854 in seinen vergleichend-morphologischen Studien an F. verna gezeigt hat). Bei den getreidekornähnlichen Bulbillen übertrifft die mit Reservestoffen (hauptsächlich Raffinose neben Saccharose: AUGSTEN 1967) gefüllte Wurzelknolle die Knospe selbst an Größe um ein Vielfaches; erst nach dem Abfallen und Austreiben wird ihre Natur augenfällig. METCALFE (1938) hat die Entwicklung der Brutknospen eingehend studiert und mit jener der basalen Wurzelknollen verglichen. Letztere unterscheiden sich von den achselbürtigen Bulbillen dadurch, dass sie von Beginn an positiv geotrop orientiert sind. In ähnlicher Weise wie die Neubildung junger Wurzelknollen mit der Entwicklung von Adventivknospen an der Grundachse in Verbindung steht, kommt es nicht selten zu vermehrter Knospenbildung auch in den Blattachseln, aus deren Parenchym sukzessiv Spross- und Wurzelinitialen entstehen können; auch an den Bulbillen können weitere Adventivknospen erscheinen und zu komplexeren Strukturen führen.

Mykorrhiza. In den Nährwurzeln wurde arbusculäre Mykorrhiza nachgewiesen.

Pollenmorphologie ${ }^{3}$. Der Pollentyp von Ficaria entspricht prinzipiell jenem von Ranunculus-Arten. Die Oberflächenornamentierung ist mikroechinat und perforat; als Aperturen finden sich Colpen. Der Pollen-Durchmesser (vgl. POGAN \& WCISŁO 1981a) liegt bei der diploiden F. calthifolia um 32-33 $\mu \mathrm{m}$, bei wohlentwickelten Pollenkörnern tetraploider F. verna im Mittel um 35-37 $\mu \mathrm{m}$. Bei letzterer Sippe ist der Pollen (entsprechend den meist schwer gestörten Meiosen der Pollenmutterzellen) relativ uneinheitlich; die Größe variiert stark (Mikro- und Makropollen); neben ,regulären“ tricolporaten Pollenkörnern kommen solche mit 4 oder mehr Aperturen vor, außerdem syncolpate sowie pantocolpate Formen $(\rightarrow$ Abb. 1). Über die Ultrastruktur und Differenzierung des Sporoderms berichtet ROLAND-HEYDECKER (1964) bzw. ROLAND (1969).

\footnotetext{
${ }^{3}$ von HeIDEMARIE HALBRITTER (Wien)
} 


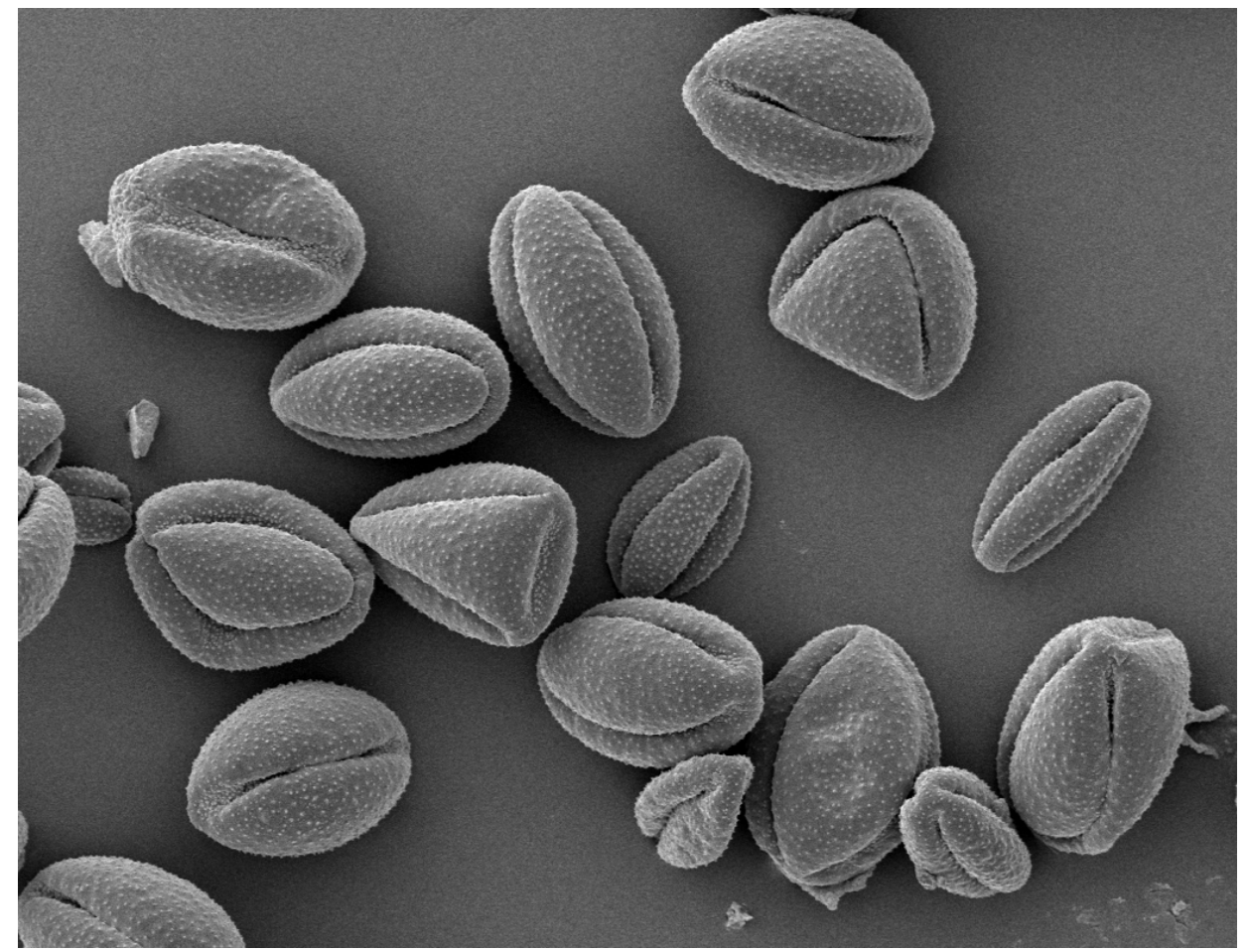

Abb. $1 \mid$ Ficaria verna s. str. Trockener Pollen im Rasterelektronenmikroskop. (Herkunft der Pflanzen: Arboretum im Botanischen Garten der Universität Wien; REM-Aufnahme: HEIDEMARIE HALBRITTER)

Genom-Analyse und Phylogenie. Bei Untersuchungen an den Sippen um Ficaria verna hat sich gezeigt, dass anhand der Genom-Größen (bzw. des DNA-Gehalts des Zellkerns) zwei Abstammungsgruppen sichtbar werden: eine westliche (mit $F$. ambigua als diploidem Vertreter) und eine östliche (mit $F$. calthifolia). Dass sich eine entsprechende Divergenz auch innerhalb der tetraploiden $F$. verna abzeichnet, hat zur Hypothese geführt, die Populationen westlich des Rheins („Western Ficaria verna lineage“) und jene Zentraleuropas („Eastern Ficaria verna lineage“) entwickelten sich voneinander unabhängig aus den jeweiligen diploiden Ausgangssippen durch Polyploidisierung (DRENCKHAHN \& al. 2017). In den Niederlanden wurden intermediäre (hybridogene) Populationen zwischen diesen beiden verna-Typen nachgewiesen.

Karyologie und Embryologie. Die Chromosomen-Grundzahl der Gattung ist $\mathrm{x}=8$. Diploide Sippen sind $F$. calthifolia, $F$. fascicularis, $F$. ficarioides sowie $F$. ambigua; Polyploidie ist bei $F$. verna s. str. $(4 \mathrm{x}, 5 \mathrm{x})$ und $F$. grandiflora $(4 \mathrm{x}, 5 \mathrm{x}, 6 \mathrm{x})$ bekannt. Triploide Pflanzen sind nach bisheriger Kenntnis als Hybriden zwischen $F$. verna und den diploiden Arten, F. ambigua bzw. F. calthifolia, zu interpretieren. Triploide und tetraploide Pflanzen eines calthifolia-Morphotyps von den Westgriechischen Inseln (DRENCKHAHN \& al. 2017) bedürfen weiterer Untersuchungen. Die karyologischen Verhältnisse wurden einerseits in Großbritannien (GILL \& al. 1972, MARCHANT \& BRIGHTON 1974, NICHOLSON 1983), andererseits in Polen eingehender studiert. Der Karyotyp des Basis-Genoms ist bei den untersuchten Sippen recht einheitlich (vgl. POGAN \& WCISŁO 1974) und unterscheidet sich deutlich von den bei Ranunculus gefundenen Karyotypen (BALTISBERGER \& HöRANDL 2016). Cytologisch verhält sich die tetraploide $F$. verna wie eine Autopolyploide, stimmt jedoch morphologisch nicht völlig mit experimentell aus Diploiden erzeugten Pflanzen überein (NICHOLSON 1983). GREEN \& THOMAS (1961) fanden in den Bulbillen der tetraploiden $F$. verna vereinzelt auch triploide Zellen.

B-Chromosomen wurden an Pflanzen sowohl aus England und den Kanal-Inseln (GILL \& al. 1972, MARCHANT \& BRIGHTON 1974) als auch aus Ungarn (POGAN \& WCISŁO 1981b) festgestellt, und zwar 
in Mischpopulationen von di- und tetraploiden Sippen. Die B-Chromosomen (1-8 akzessorische Chromosomen) kommen allerdings nur bei den Diploiden vor (desgleichen auch in österreichischem Material: J. GREILHUBER, mündl. Mitt.), beeinflussen aber weder die Fertilität noch die Wuchskraft der Pflanzen nennenswert.

Die Mikrosporogenese verläuft bei diploiden Sippen normal. Die Embryosackentwicklung erfolgt nach dem verbreiteten Polygonum-Typ zu einem 8- oder (nach Verschmelzung der Polkerne) 7-kernigen Embryosack; bei einem abgewandelten Typ degeneriert während der Megasporogenese die mikropylare Dyade bereits vor der 2. meiotischen Teilung (POGAN \& WCISŁO 1981a). Die Ausbildung des triploiden, nucleären Endosperms setzt nach der Befruchtung schon vor der Weiterentwicklung des Embryos ein; die Samenanlage ist anatrop, unitegmisch und crassinucellat. METCALFE (1939) beobachtete an emaskulierten Pflanzen auch bei fehlender Bestäubung vereinzelt Samenentwicklung mit normal erscheinenden Embryonen; er vermutete daher Apomixis, ohne dies embryologisch zu belegen.

Während bei F. calthifolia nach experimenteller Bestäubung durchwegs die Samenentwicklung rasch einsetzte, konnten WCISŁO \& POGAN (1981) bei F. verna s. str. (deren Embryosäcke in der Regel abortieren) zwar mehrfach erfolgreiche Befruchtung nachweisen, doch führte dort die stark verzögerte Weiterentwicklung nur bei $1 \%$ der Karpelle zu Samen mit Endospermgewebe und einem mehrzelligen Embryo.

Blüten- und Ausbreitungsökologie. Die Blüten sind in der Regel zwittrig und schwach protandrisch. Gynomonözie und Triözie wurden vereinzelt beobachtet. Detaillierte Untersuchungen zur Bestäubung führte MARSDEN-JONES $(1935,1937)$ in England an F. verna durch. Die äußeren Staubblätter (mit extrorsen Antheren) entlassen den Pollen noch bevor die Karpellnarben empfangsbereit sind. Pollen und Nektar werden für kurzrüsselige Insekten dargeboten; deren Besuch ist für eine erfolgreiche Samenentwicklung unabdingbar, da die Pflanzen weitestgehend selbststeril sind (WOLTER 1933, MARSDEN-JONES 1937, POGAN \& WCISŁO 1981a). Bei fehlender Kreuzbestäubung kann es zwar zu Selbstbestäubung kommen, da sich die zuletzt öffnenden, inneren Staubblätter über die Karpelle beugen; sie führt jedoch nur zu geringem Samenansatz. MARSDEN-JONES konnte an Ficaria-Blüten mehr als 80 Arten von Coleopteren, Hymenopteren, Lepidopteren und Dipteren beobachten. Die wichtigsten Pollenüberträger waren Glanzkäfer der Gattung Meligethes (Nitidulidae). Auch WOLTER (1933) beobachtete (um Breslau, möglicherweise an F. calthifolia) den Blütenglanzkäfer Meligethes nigrescens [= M. picipes] als Bestäuber. Andere sehr häufige Blütenbesucher sind Bienen (Apis mellifera). Dementsprechend registrierte auch ANDREAS (1954) vor allem Bienen und Blütenglanzkäfer als Besucher.

F. verna gilt als myrmekochor (SERNANDER 1901, BRESINSKY 1963); die ausgereiften Nüsschen besitzen als Elaiosom eine verlängerte, zunächst fleischige (vertrocknet längs gefurchte) Basis, in der Fette, Stärke, Zucker und Vitamin C angereichert sind.

Vegetative (klonale) Ausbreitung erfolgt durch Fragmentierung der Grundachse und des Wurzelknollen-Büschels in Einheiten mit wenigstens einer Erneuerungsknospe; die schlafenden Knospen einzelner Wurzelknollen können dann nach der Sommerruhe beginnen, sich zu neuen Pflanzen zu entwickeln. Bei den bulbillenproduzierenden Sippen fungieren die stängelständigen, nach dem Verwelken der Stängel verselbständigten Brutknospen als außerordentlich effiziente Ausbreitungseinheiten (siehe unten). Die weitestgehend klonale Fortpflanzung bedeutet aber nicht notwendigerweise auch eine eingeschränkte Diversität: gemessen am Verhältnis unterschiedlicher Genotypen war etwa die klonale Diversität der Ficaria-Populationen intensiv genutzter Mähwiesen wesentlich höher als jene der Populationen benachbarter Waldbereiche (REISCH \& SCHEITLER 2009). Die gelegentliche massenweise Verschwemmung 
von Bulbillen wie von Wurzelknollen ist Anlass der Berichte vom „Getreideregen“. GAMS (1932) spricht von $F$. verna (in Zusammenhang mit ,amphibisch“ wachsenden Beständen) auch als einer Hydrochoren.

Standortsökologie. In Wuchsform (Wurzelknollen-Geophyten mit monokarpen Blühsprossen; lediglich die abgeleitete $F$. verna s. str. zeigt manchmal Übergänge zu hemikryptophytischer Überdauerung) und Wuchsrhythmik (ephemeroide Frühjahrsblüher mit ausgeprägter Sommerruhe) sind sämtliche Arten der Gattung an mediterrane Klimabedingungen angepasst; die Vorkommen liegen schwerpunktsmäßig in sommergrünen Laubwäldern bzw. deren heliophilen Saumgesellschaften.

Inhaltsstoffe. Toxisch ist der auch bei Ranunculus-Arten verbreitete cyclische Kohlenwasserstoff Protoanemonin $\left(\mathrm{C}_{5} \mathrm{H}_{4} \mathrm{O}_{2}\right)$, ein mit der Entwicklung der Blüten gebildetes Lacton (Reizung von Schleimhäuten, Nieren und Harnwegen; bei größerer Menge auch Lähmungserscheinungen). In den Wurzelknollen wurden Triterpen-Saponine (Glucoside von Hederagenin und der Oleanolsäure) nachgewiesen (vgl. z. B. MARSDON \& al. 2006). Ascorbinsäure wurde vor allem aus den Chloroplasten isoliert. An organischen Säuren wurde mengenmäßig überwiegend Citronensäure gefunden. Als Blütenfarbstoffe sind Carotinoide (Xanthophyll) und Flavonoide nennen (zu letzteren vgl. etwa TOMCZYK \& GUDEJ 2003).

Nutzen und Verwendung. Der von den alten Kräuterbuch-Autoren stammende deutsche Name weist bereits auf die frühere Verwendung bei Skorbuterkrankungen (Skorbut $=$ Scharbock) hin, die durch Vitamin-C-Mangel hervorgerufen werden. Die Laubblätter von Ficaria verna gelten als vitaminreich; das (junge!) Laub wird auch heute von Wildkräuter-Liebhabern als Salat oder Gemüse verwendet, sollte aber nur vor dem Erscheinen der Blüten geerntet werden (vgl. oben!). Die Speicherwurzeln wurden auf Grund der Signaturenlehre früher als Mittel gegen Feigwarzen und Hämorrhoiden verwendet; Extrakte werden mittels Salben auch heute bei Hämorrhoidalbeschwerden und Rosacea appliziert (als möglicher Wirkstoff gelten Saponine) oder in der Kosmetik genutzt. Möglicherweise wurden die Speicherwurzeln (ob ihres Gehalts an Reservestoffen) bereits in prähistorischer Zeit auch als Nahrungsmittel genutzt, wie das gehäufte Vorkommen verkohlter Wurzelknollen zusammen mit anderen gesammelten Vegetabilien an neolithischen Wohnplätzen Nordwesteuropas vermuten lässt (KLOOSS \& al. 2015).

Zierpflanzen. Als gärtnerisch interessante Frühblüher sind Ficaria verna und verschiedene ihrer dekorativen Varianten seit langer Zeit beliebt (vgl. HARPER 1999), vor allem in England, wo in Gartenkatalogen am Ende des 18. Jahrhunderts an die 800 verschieden benannte Cultivaria angepriesen wurden! Auch heute werden noch zahlreiche Sorten angeboten (vgl. z. B. MATTERN 1995). Hier seien beispielhaft genannt solche mit unterschiedlich ornamentiertem, braunviolett, schwarz, gelb ('Hoskin's Variegated') oder silbern ('Brambling') geflecktem oder überhaupt schokoladenbraun überlaufenem ('Brazen Hussy') Laub; ebenso auffallend orange- oder cremefarben bis weiß blühende ('Cupreus', 'Randall's White') Klone; beliebt sind auch Pflanzen mit gefüllten ('Colarette', 'Pleniflora') oder vergrünten ('Green Petal') Blüten. Als Zierpflanzen eingeführt wurde auch die großblütige (bis über $5 \mathrm{~cm} \varnothing$ ) $F$. grandiflora [Ranunculus ficaria subsp. ficariiformis] aus dem Mittelmeergebiet, die z. B. auf den Britischen Inseln häufig verwildert vorkommt; neuerdings wird auch $F$. calthifolia im Handel angeboten.

Krankheiten und Schädlinge. Als Beispiele parasitärer Pilze seien angeführt: Rostpilze: Uromyces ficariae (SCHUMACH.) LÉV. (Uredo- und Teleutosporenlager als braune bis dunkelbraune Pusteln auf beiden Blattseiten und auf Blattstielen; kein Wirtswechsel), U. poae RABENH. (Pyknidien auf Blattoberund orange Aecidien auf Blattunterseiten und -stielen, befallene Blattflächen schwielenartig deformiert; Wirtswechsel mit Poa-Arten), U. rumicis (SCHUM.) WINT. (Pyknidien auf Blattober- und orangefarbene Aecidien auf Blattunterseiten sowie Blattstielen, befallene Blattflächen kaum schwielenartig deformiert; 
Wirtswechsel mit Rumex-Arten); Brandpilze: Entyloma ficariae FISCH. v. WALDH. (kleine Sori in Blättern, verursacht kleine, gelbe bis gelbbraune Flecken; die nahverwandte E. majewskii VÁNKY \& M. LUTZ wurde jüngst aus dem Iran beschrieben); Urocystis ficariae (LIRO) MOESZ (dunkelbraune Sporenmassen in blasenförmigen, silbrigen Anschwellungen an Blättern, Blattstielen und Stängeln); der Falsche Mehltau Peronospora ficariae L.R. TUL. ex MOESZ (an Blättern; befallene Blätter stark gewölbt, Oberseite graugrün, Unterseite von dichtem, grauweißem bis grauviolettem Konidienträgerrasen bedeckt) ${ }^{4}$. Als Phytophage auf Ficaria verna werden die Larven des Wicklers Olindia schumacherana (FABR.) genannt.

Volksnamen. Die im folgenden angeführten Vernakularnamen [nach MARZELL 1977] beziehen sich größtenteils, wenn nicht ausschließlich auf $F$. verna s. str. Die fettglänzenden Blüten haben der Art ähnliche Namen wie dem Ranunculus acris verschafft, so Botterblom (niederdeutsch), Botterschlöckelche (Heinsberg/Niederrhein), Anke(n)blüemli (Anke: ,Butter') (Schweiz), Schmalzblume (vielfach), Schmelzkächele (Bopfingen), (auch: Butter-)Schmirgel (Nordböhmen), Fettpfandl (Steiermark), Glisserli, Glitzerli (Schweiz), Spiegelblome (Bremen), Goldblümchen (um Jena). Nach den sternförmigen Blüten: Sterne(n), Sterneli (Schweiz), Sternblum (z. B. Oberbayern), Sterne(n)blüemli (Graubünden, Zug). Nach der rundlichen Gestalt der Laubblattspreite heißt die Art auch Pfennigkraut (rheinisch), Penningskraut (Dillkreis), Pennigblaume (Windheim/Minden), Kreuzerlan, Kreizerblatlan (Kärnten). Die länglichen (feigwarzen- oder hodenähnlichen) Speicherwurzeln veranlassten schon die alten Kräuterbuch-Autoren zu Namen wie Fickwartenkrut (BRUNSCHWYG 1500: ,darumb das sin wurtzel geschaffen ist glich den fickwartzen“), Feigblatternkraut (FUCHS 1543), bzw. Pfaffenhödlin (BRUNFELS 1532), Biberhödlin, Rammenhödlin (BoCK 1539). Die getreidekorngroßen Brutknospen veranlassten Namen wie Erdtgersten (V. CORDUS 1561), Himmelsgerste (Posen, Liegnitz). Auf die Blütezeit beziehen sich Maienkraut (Jena), Maiblä(d)r (Altmark), Osterblume (Murnau/Oberbayern), Uffartsblüemli (Uffart ist der Himmelfahrtstag, an dem sich die Mädchen mit Kränzen aus diesen Blumen schmückten) (Saas/Graubünden), Herrgottsblüemli (St. Gallen). Das junge Laub wurde als Salat gegessen: Pennikzelot [Pfennigsalat] (Kinheim/Wittbich), Zigeunersalat (Kärnten), Henkel(H inkel-) zaloat (Siebenbürgen), Mörkelnschlot [-salat] (Kleinenbroich/M.-Gladbach), Kassen[Kressen-]kraut (Zerpenschleuse/ Niederbarnim). Nach ihrer Verwendung als Heilpflanze gegen „Scharbock“ (Skorbut) Scharbockskraut (E. CoRDUS 1534), Scharbuck (Kaltenkirchen/ Mittelholstein), Schoorbock (Hannover, Lüneburg). Manche Namen sind Übertragungen von oberflächlich ähnlichen Pflanzen, so Gäl's [gelbes] Geißblüemli (das weiße ist Anemone nemorosa) (St. Gallen), Lütt-Mümmelkenbläder (Mümmelken = Nuphar luteum) (Mecklenburg). Sonstige Bezeichnungen sind ferner Eierkraut (Gaussig/ Bautzen), Gause[Gänse-]blömkes (Westfalen), Pelterchen, Pülterchen (Mosel, Nahe).

Volkstümlich-Historisches. Mit dem Volksnamen „Himmelsgerste“ verbunden sind Berichte vom Getreideregen. Dazu seien aus GOEPPERT (1831: 559-560) Beobachtungen zitiert, die „zu den Sagen von Getreideregen Veranlassung gegeben haben ... “: „Professor v. Jacquin gedenkt in einem Schreiben an den Ob. Med. Rath Klaproth zu Berlin, vom 23. December 1803, eines Knollenregens in Ober-Oestreich im Sommer jenes Jahres. »Eine genaue Untersuchung des dort angeblich vom Himmel gefallenen Korns lehrte indessen bald, daß das sogenannte Korn Knollen vom kleinen Schöllkraute (Ranunculus Ficaria) waren, welche Regengüsse losgeschwemmt und auf niedriger liegende Wiesen und Felder gebracht hatten. Uebrigens sei diese Erscheinung nicht ganz neu, sondern habe sich schon mehreremale in Ober-

${ }^{4}$ Zusammenstellung ergänzt von HERMANN VOGLMAYR (Wien) 
Oestreich und anderen Gegenden zugetragen, daher man auch das kleine Schöllkraut Erdgerste zu nennen pflegte.« Im Anfang dieses Jahrhunderts, im Juli 1805, ereignete sich etwas Aehnliches an mehreren Orten Schlesiens, nämlich zu Landeshut, Hansdorf, Bärsdorf, Tannhausen, Voigtsdorf bei Grottkau, Strehlen und Nimptsch. Es fanden an diesen Orten angeblich Getreideregen statt, die eingeschickten Körner waren aber, nach dem Urtheil des Medicinal-Assessor Hrn. Dr. Günther, nichts anderes als die Wurzeln unserer Pflanze.“

Hybriden. Über Hybriden ist wenig Konkretes bekannt. Der Bastard $F$. calthifolia $\times F$. verna wurde in Polen (POGAN \& WCISŁO 1983, 1986) und Österreich (J. GREILHUBER, unveröff.) karyologisch untersucht. Eine detaillierte Untersuchung solcher Hybriden (beschrieben als $F$. ×sellii DUCHOSLAV \& al.) in Mähren und der Slowakei zeigte ihr regelmäßiges Auftreten im Bereich sympatrischer Vorkommen der Parentalarten auf (ŠIKOVÁ 2014, POPELKA \& al. 2019). Von den Britischen Inseln ist $F$. ambigua $\times$ $F$. verna mehrfach nachgewiesen worden (vgl. dazu JONES 1975). Beide Hybrid-Kombinationen sind hochgradig sterile Triploide.

\section{Bestimmungsschlüssel zu den Arten ${ }^{5}$}

1 Jahressprosse gestaucht (höchstens das basale, unterirdische Internodium 1-4[-7] cm lang), Laubblätter daher alle \pm rosettig gehäuft, Blattachseln stets ohne Brutknospen, Blütenstiele direkt der Rosette entspringend (selten ein zweites kurzes, bodennahes Internodium unter dem Blütenstiel entwickelt), in der Regel blattlos (gelegentlich mit einem Stängel- oder Hochblatt), zur Blütezeit aufrecht, meist 5-8(-10) cm lang, fruchtend zum Boden hinabgekrümmt, Nüsschen durchwegs wohl entwickelt 2. F. calthifolia

1* Jahressprosse mit \pm gestreckten Internodien, Laubblätter daher kaum rosettig gehäuft, Blühtriebe aus aufrechtem Grund schwach aufsteigend bis fast horizontal ausgerichtet, nicht selten verzweigt, bis ca. $25 \mathrm{~cm}$ lang, mit (zumindest 2) \pm gestreckten Internodien und in der Regel mit 2 oder mehr Stängelblättern, Blütenstiel zur Fruchtzeit nicht zum Boden gekrümmt

2 Stängelblattachseln (zumindest nach dem Abblühen) mit elfenbeinfarbenen Brutknospen (Bulbillen), Sammelfrucht mit größtenteils verkümmerten Nüsschen (selten mehr als 6 entwickelt, auch diese meist nicht keimfähig), Stängelblätter in der Regel auch in der oberen Hälfte des Blühsprosses, Blütendurchmesser meist ca. 2-21/2 cm, Kronblätter in der Regel nur bis $5 \frac{1}{2} \mathrm{~mm}$ breit, sich nicht oder wenig überdeckend, Staubblätter selten mehr als 35, Pollen stets irregulär ausgebildet (Pollenkörner teilweise leer), Schließzellen der Spaltöffnungen mit ca. 24-28 Chloroplasten (Mittelwerte) 1a. F. verna s. str.

2* Stängelblattachseln auch nach der Blütezeit ohne Brutknospen, Sammelfrucht mit zahlreichen wohlentwickelten, keimfähigen Nüsschen, Stängelblätter meist auf das untere Drittel des Blühsprosses beschränkt, Blütendurchmesser 2-4 $\mathrm{cm}$, Kronblätter 4-9 $\mathrm{mm}$ breit, einander überdeckend, Staubblätter 20-50, Pollen normal entwickelt, Schließzellen der Spaltöffnungen mit ca. 13-17 Chloroplasten (Mittelwerte)

1b. F. ambigua

\footnotetext{
${ }^{5}$ Die Angaben zu der im Gebiet bisher nicht sicher nachgewiesenen $F$. ambigua wurden mangels ausreichender eigener Erfahrung mit Pflanzen am natürlichen Standort im Wesentlichen der Literatur entnommen. Die Daten (Mittelwerte) zu den Spaltöffnungs-Schließzellen hat NicHOLSON (1981) an britischem Material erhoben.
} 


\section{Ficaria verna ${ }^{6}$}

HUDSON 1762, Fl. Angl. 214, nom. nov. pro: Ranunculus ficaria LINNAEUS 1753, Sp. P1.: 550 (quoad lectotyp. ${ }^{7}$ ). - Syn.: Ficaria ranunculoides RoTH 1788, Tent. Fl. Germ. 1: 241, nom. illegit. (superfl. pro Ficaria verna HuDS.); F. verna var. bulbifera NOULET 1837, Fl. Bass. Sous-Pyr.: 16; F. ranunculoides var. divergens F. W. SCHULTZ 1855, Arch. Fl.: 122; Ranunculus ficaria var. divergens (F. W. SCHULTZ) HORW. 1933 in HORWOOD \& NOEL, Fl. Leicester: 19; R. ficaria var. cordifolius NEILR. 1859, Fl. Nieder-Oesterreich: 685; R. ficaria var. bulbifer AlBERT 1908 in AlBERT \& JAHANDIEZ, Cat. Pl. Vasc. Var: 7; R. ficaria var. sinuatus HoRw. 1916, Rep. Bot. Exch. Club. Brit. Isl. 4: 312; R. ficaria var. bulbifer MARSDEN-JONES 1935, J. Linn. Soc. London (Bot.) 50: 40, nom. illeg. (non [三] ALBERT 1908); R. ficaria subsp. bulbifer LAWALRÉE 1955 in ROBYNS (Ed.), Fl. Gén. Belgique, Sperm. 2: 600, nom. illeg. (non [三] R. ficaria var. bulbifer ALBERT 1908); Ficaria verna ,agsp.“ bulbifera Á. LövE \& D. LÖVE 1961, Bot. Not. 114: 52, nom. illeg. (non F. verna var. bulbifera NOULET 1837); Ficaria bulbifera HOLUB 1961, Preslia 33: 400; Ranunculus ficaria subsp. bulbilifer LAMBINON 1981, Bull. Jard. Bot. Natl. Belgique 51: 462. - Frühlings-Scharbockskraut, Gewöhnliches Sch., Knöllchen-Sch. Engl.: lesser celandine; dän.: almindelig vorterod; niederl.: gewoon speenkruid; franz.: ficaire; ital.: ficaria à bulbilles; slowen.: spomladanska lopatica; ungar.: salátaboglárka; slowak.: blyskáč cibul'katý; tschech.: orsej jarní; sorb.: bórliški; poln.: ziarnopłon wiosenny; litau.: pavasarinis švitriešis; russ.: чистяк весений [čistjak vesennij]. - Abb.: $\rightarrow$ DRENCKHAHN 2016, Abb. 9, 12, http://www forum-geobotanicum.net/articles/vol 7-2016/drenckhahn ficaria/drenckhahn ficaria calthifolia.html

Pflanze ausdauernd (Wurzelknollen-Geophyt, nicht selten auch hemikryptophytisch unter der Laubstreu überdauernd), Wuchshöhe meist 8-15(-20) $\mathrm{cm}$. Wurzelstock (Grundachse) extrem verkürzt, mit einem Bündel mäßig zahlreicher (3-10[-15]) \pm birn- bis keulenförmiger, meist 1-4(-7) cm langer, bis etwa $7 \mathrm{~mm}$ dicker Speicherwurzeln und einem Kranz jährlich neugebildeter, dünner $(<1 \mathrm{~mm})$, verzweigter Nährwurzeln. Jahress pross frühjahrsgrün, nicht selten vom Grund an verzweigt, Luftsprosse mehrgliedrig, mit einigen oberirdischen Stängelinternodien (häufig über einem $1 / 2-3[-5] \mathrm{cm}$ langen basalen Internodium im Boden oder in der Laubstreu), aus aufrechtem Grund schwach aufsteigend bis fast horizontal ausgerichtet, insgesamt bis ca. $30 \mathrm{~cm}$ lang. Grundständige Blätter wenige: meist 2-3 lanzettlich- bis eiförmig-dreieckige, $1(-4) \mathrm{cm}$ lange, scheidenartige, häutige (oft hinfällige) Niederblätter und 1(-2) lang gestielte, am Grund scheidig verbreiterte Laubblätter; am bodennahen Sprossknoten meist 2 weitere lang (bis $12 \mathrm{~cm}$ ) gestielte Laubblättter; Stängelblätter wenigstens 2 (bis 4 oder mehr), nach oben zu kürzer gestielt und kleiner, die beiden letzten und die Vorblätter der Seitensprosse oft scheinbar gegenständig, stets mit zumindest postfloral wohlentwickelten, zuletzt bis $6 \times 4 \mathrm{~mm}$ großen Brutknollen (Bulbillen) in den Blattachseln. Blattspreiten meist eiförmig bis rundlich (oder fast nierenförmig), vorn stumpf gerundet, mit herzförmigem Grund und meist offener $\Lambda$-förmiger bis verschmälerter Bucht, die Mehrzahl wenig länger als breit (größte Spreiten ca. 21/2-41/2 $\times 2 \frac{1}{2}-4 \mathrm{~cm}$ ); Blattrand seicht und entfernt gekerbt bis angedeutet lappig (insbesondere oberste Stängelblätter oft schwach 7- bis 3-lappig). Blüten (eines Jahressprosses) meist 1-3 (selten mehr), endständig an den beblätterten Luftsprossen (seitliche oft auch blütenlos endend); Blütenstiele postfloral \pm gerade gestreckt bleibend. Äußere Blütenhüllblätter (Kelchblätter) 3(-5), breit eiförmig, $(5-) 6 \frac{1}{2}-8(-9) \times 3 \frac{1}{2}-4(-5) \mathrm{mm}$, aus häutigem Grund krautig und zumindest in den vorderen Dritteln grün. Innere Blütenhüllblätter (Kronblätter) (6-)8-10(-14), (oft auch schmal) elliptisch (-eiförmig), (9-)10-14(-16) × 31/2-51/2(-61/2) mm; Nektar-

\footnotetext{
${ }^{6}$ lat. vernus »zum Frühling gehörig« (Adjektivbildung zu lat. ver »Frühling«).

${ }^{7}$ L. Benson 1954, Amer. Midl. Naturalist 52 : 369.
} 
gruben $0,8-1,0 \mathrm{~mm}$ lang. Staubblätter selten $>35$. Fruchtblätter meist $15-25$, aber keine oder nur wenige (selten > 6) heranreifend; voll entwickelte Nüsschen (einschl. der stielartig verschmälerten Basis) ca. $3 \mathrm{~mm}$ lang und 2(21/4) mm dick, kugelig gewölbt, nur schwach gekielt, \pm locker kurz behaart, Griffelspitze undeutlich (bis 0,1 mm). - Chromosomenzahl: $2 \mathrm{n}=32$ (selten auch 40: ANDERS-GASSER 1985). - Blütezeit: III-V.

Standort und Vergesellschaftung. In Mitteleuropa gesellig in Auwäldern und krautreichen Laubwaldgesellschaften auf nährstoff- und basenreichen, in der Regel grundwasser- oder sickerfeuchten, humosen, stets einigermaßen tiefgründigen Böden (gemieden werden ausgesprochen bodensaure Substrate), von der Ebene bis in die montane Stufe (ausnahmsweise bis 1800 m, wohl durch Almwirtschaft verschleppt); auch in entsprechenden Waldsäumen, Gebüschen oder Hecken, in Gärten (im Regentrauf der Obstbäume: BRAUN-BLANQUET 1924) und randlich in (zumindest im Frühjahr) bodenfeuchten bis vernässten Nutzwiesen; in der Aulandschaft auch in Überschwemmungsbereiche vordringend (vgl. dazu auch GAMS 1932). Nach OBERDORFER (2001) schwache Querco-Fagetea-Art; in verschiedenen Edellaubwäldern (Fagetalia-sylvaticae-Gesellschaften), als konstanter Begleiter vor allem in AuwaldGesellschaften des Alnion incanae, auch in thermophilen Saumgesellschaften des Galio-Alliarion und frischen Fettwiesen-Gesellschaften des Arrhenatherion.

Allgemeine Verbreitung. Fast ganz Europa; fehlt im nördlichen Fennoskandien sowie in weiten Teilen der Iberischen Halbinsel und Griechenlands; im Mittelmeergebiet selten und meist nur in den Gebirgen. [Karte: TAYLOR \& MARKHAM 1978: 1016; vgl. dazu JALAS \& SUOMINEN 1989: 186, no. 1835]. In Irland vermutlich nicht autochthon, ebensowenig auf Korsika; auf den Färöer eingeschleppt. In Island als Zierpflanze eingeführt, ebenso in Nordamerika (S-Kanada, USA, hier zumindest seit dem Ende des 19. Jh. nachgewiesen [älteste Aufsammlung von 1891: POST \& al. 2009]; von Missouri, Tennessee und Virginia bis S-Ontario, S-Quebec und Neufundland, im Westen von N-Oregon bis Brit. Columbia, im Süden auch in Texas; gebietsweise als invasives Unkraut bekämpft; vgl. auch AXTELL \& al. 2010, KERMACK \& RAUSCHERT 2019) und Australien (Victoria, selten).

Zonale Arealdiagnose: sm-temp-(b)·oz1-3 EUR

Regionale Arealdiagnose: submed-(pont)-me-scot-(scand)

Verbreitung im Gebiet. In allen Ländern des Gebiets weit verbreitet und häufig (nur in den inneren Alpentälern teilweise fehlend).

Lebensgeschichte. $F$. verna s. str. tritt im zeitigen Frühjahr vielerorts in großen Herden und daher aspektbildend auf; entsprechend ihrer klonalen Vermehrung bildet sie im Frühjahr nicht selten deckende Blatt-Teppiche. Den Lebenszyklus haben zuletzt KRIŽO \& KORÍNEKOVÁ (1987) eingehender studiert. Nach der aktiven Vegetationszeit mit reichlicher Belaubung im beginnenden Frühjahr und der rasch darauf folgenden Blütezeit sterben die monokarpen Luftsprosse bereits im Frühsommer ab; die ausgeprägte Ruhezeit findet erst im September ihr Ende, wenn sich basal an der (oder den) im vorjährigen Herbst gebildeten Erneuerungsknospe(n) der Grundachse reichlich neue Nährwurzeln bilden. In den Achseln der äußeren, das Spross-Primordium einhüllenden Niederblätter entwickeln sich an ruhenden Achselknospen die knollenförmigen Speicherwurzeln, welche diese häutigen Hüllorgane teilweise seitlich durchbrechen; in den Achseln der inneren Niederblätter entstehen die Erneuerungsknospen für die nächste Vegetationsperiode. In der Folgezeit werden die Blatt-Primordien (samt den Achsel-Bulbillen der Stängelblätter) angelegt. Im Laufe des Novembers differenzieren sich schließlich die Blütenorgane, wobei die Kelchblätter auch die zuletzt differenzierten Bereiche des Androeceums und Gynoeceums 
einhüllen, so dass noch im Spätherbst zumindest die Anlage der generativen Organe vollzogen ist. Nach der durch den Winter erzwungenen Wachstumspause beginnt Februar/März mit der Vergrößerung der sporogenen Zellen die Mikro- und Megasporogenese sowie die aktive Vegetationszeit der neuen Saison.

Mikro- und Megasporogenese sind bei F. verna s. str. in hohem Maße gestört (WCISŁO \& PoGAN 1981). Der Pollen degeneriert in beachtlichem Maße, selbst funktionsfähige Pollenkörner sind genetisch nicht ausbalanciert. Der Anteil gut entwickelten Pollens beträgt nach POGAN \& WCISŁO (1981a) meist nur 30-65 \%; ein von KRÍZO \& KORÍNEKOVÁ (1987) durchgeführter Test zur Keimfähigkeit erbrachte Werte von $25-90 \%$ (doch bezieht sich letzterer Wert wohl auf die diploide $F$. calthifolia, die in den Analysen von 10 Populationen eingeschlossen war, aber nicht differenziert beurteilt wurde). Die slowakischen Autoren berichten auch über häufige Anomalien in der Embryosackentwicklung; dem entspricht die bekannte Tatsache, dass sich bei $F$. verna nur eine geringe Anzahl der Fruchtblätter überhaupt weiter entwickelt (2-3\% nach MARSDEN-JONES 1935). Dabei degenerieren auch in anscheinend gut entwickelten Nüsschen 7-kernige Embryosäcke, manche enthalten nur mehr das Endosperm (POGAN \& WCISŁO 1981a); lediglich einzelne Samen sind überhaupt zur Keimung fähig. Tests mit 50 Individuen ergaben, dass bei $76 \%$ ihrer Blüten sämtliche Karpelle abortierten, und dass für die wenigen entwickelten Nüsschen (3\% der Versuchsreihe mit insgesamt über 3000 Karpellen) die Keimrate nur $18 \%$ betrug (gegenüber $>70 \%$ bei F. ambigua: MARSDEN-JONES 1935). Sämlinge entwickeln nach IRMISCH (1854) in der Keimblatt-Scheide eine erste Knospe mit der zugeordneten Wurzelknolle; diese allein überdauern die folgende Sommerruhe, während Keim- und ein allenfalls gebildetes Laubblatt absterben. Blühreife dürfte erst nach mehreren Jahren bei entsprechender Erstarkung und Ausbildung eines mehrgliedrigen Wurzelknollen-Büschels erreicht werden.

Die Vermehrung erfolgt bei der F. verna s. str. aber fast ausschließlich vegetativ und mit großer Effektivität über die blattachselbürtigen Bulbillen (vgl. oben: Morphologie). Wie die Wurzelknollen sind sie einer obligaten Sommerruhe unterworfen, beginnen ebenfalls bereits im Herbst (grundsätzlich aber etwas später als jene: AUGSTEN 1957) zu treiben, wobei knapp $10 \%$ als „Trotzer“ zumindest eine weitere Periode im Ruhezustand verharren (WINKLER 1926). Experimente haben gezeigt, dass die Einwirkung niedriger Temperaturen nach der Sommerruhe nicht nur die winterliche Ruhezeit verkürzt, sondern die weitere Entwicklung der Pflanzen deutlich beschleunigt (MUDRACK 1935; AUGSTEN 1957 spricht von „Vernalisation“), während gleichbleibend höhere Temperaturen die Knospenentfaltung hemmen. Eine ausreichende Kälteeinwirkung ist also wesentlich für das Ergrünen und die Weiterentwicklung im Frühjahr (MUDRACK 1935, AUGSTEN 1957, COURDUROUX 1966). Nach dem Austreiben entwickeln sich die Jungpflanzen außerordentlich schnell, so dass sie teilweise schon im ersten Jahr zur Blüte kommen können und ihrerseits reichlich Bulbillen produzieren; ein Experiment erbrachte innerhalb eines Jahreszyklus 195 blühende Nachkommen von 10 Mutterpflanzen (vgl. MARSDEN-JONES 1935). $\mathrm{Zu}$ sexueller Fortpflanzung kommt es offenkundig nur ausnahmsweise; auch in Experimenten mit Pflanzen, deren Bulbillen entfernt und die künstlich bestäubt worden waren, konnte keine erfolgreiche Samenproduktion erzielt werden (KINDLER 1914, MARSDEN-JONES 1935). In Halle/Saale werden aber ganz regelmäßig einige, an den umgekehrt herzförmigen Keimblättern leicht zu erkennende, Keimpflanzen gefunden (E. JÄGER briefl.). Wie bei anderen Ficaria-Sippen kommt es außerdem zur Vermehrung durch Fragmentierung der gestauchten Grundachse und des Wurzelknollen-Büschels.

Nomenklatur. Die in Europa weitverbreitete bulbillentragende Sippe wird hier (in Übereinstimmung mit neueren Arbeiten; vgl. DRENCKHAHN 2017) als eigene Art behandelt. Sie wurde in den vergangenen Jahren (sofern eigens unterschieden) zumeist als Ranunculus ficaria subsp. bulbifer oder (seit 1981) als subsp. bulbilifer bezeichnet. Während LAWALRÉE (1955) vorsichtigerweise für seine diploide bzw. 
tetraploide Unterart jeweils Namen vergeben hatte, ohne ein Autonym zu verwenden, bezog TUTIN (1964 in der »Flora Europaea«, so auch schon vorher 1962 in der 2. Auflage der »Flora of the British Isles «) den Namen R. ficaria subsp. ficaria auf die in England verbreitete, fertile diploide Sippe (heute: F. ambigua). SELL (1994) hat diesen Gebrauch formalisiert, indem er die früheste Typisierung des linnaeischen Namens (BENSON 1954) verwarf und einen Typus vorschlug, welcher vermutlich der diploiden Sippe entspricht. Nach den Nomenklaturregeln darf ein (früher bestimmter) Lectotypus jedoch nur dann verworfen werden, wenn er in schwerwiegendem Widerspruch (,serious conflict") mit dem Protolog steht, was bei dem von BENSON gewählten Beleg (LINN 715.12) sicher nicht der Fall ist (LÆGAARD 2001). Die 3 Blühtriebe auf diesem Bogen des Linné-Herbars zeigen zwar noch keine Bulbillen, gehören aber allem Anschein nach zu der in Schweden allein vorkommenden, LINNÉ selbst wohlvertrauten Sippe.

Variabilität der Art, ähnliche Taxa. Ficaria verna variiert insbesondere hinsichtlich der Blattform (Kerbung bzw. Lappung des Blattrands, Form der Basalbucht), Fleckung oder Überfärbung der Blattspreite sowie Blütengröße und -farbe (vgl. oben: Zierpflanzen). Solche Formen sind durch die klonale Vermehrung manchmal auffällig, ihr taxonomischer Wert ist jedoch fragwürdig (so ist die als „Ranunculus ficaria var. aurantiacus" TURRILL beschriebenen Variante mit orangefarbenen Kronblättern gleichbedeutend mit cv. 'Cupreus' ${ }^{8}$ - z. B. nicht erbfest). Hinsichtlich der Blütengröße ist zu beachten, dass die Endblüte eines Jahressprosses gewöhnlich größere Kronblätter aufweist als die Folgeblüten und dass männliche Blüten größere, weibliche Blüten geringere Abmessungen zeigen als Zwitterblüten. Die mögliche biologische Relevanz solcher aberranter „micrantha“-Typen diskutierte ANDREAS (1954). Entsprechende rein weibliche Pflanzen bewahrten jedoch in folgenden Jahren hinsichtlich ihres Geschlechts keine Konstanz (MARSDEN-JONES 1935).

Die mediterran verbreitete Ficaria grandiflora ROBERT [F. verna subsp. ficariiformis (F.W. SCHULTZ) B. WALLN. ${ }^{9}$ ] gehört wie $F$. ambigua und $F$. calthifolia zum Formenkreis der $F$. verna und unterscheidet sich von diesen im Wesentlichen durch größere Dimensionen. Die robuste Pflanze entwickelt beblätterte Luftsprosse, oft 6-8 cm lange, Caltha-ähnliche Blattspreiten, Blüten mit einem Durchmesser von ca. 3-5 $(-5,7) \mathrm{cm}$ und durchwegs breiteren Kronblättern (mit elliptischen, bis 1,7 mm langen Nektargruben). Die Pflanzen fruchten gewöhnlich gut, entwickeln aber in der Regel zusätzlich Bulbillen in den Stängelblattachseln. Solche Populationen kommen, dem Gebiet zunächst, in Istrien und auf den Inseln des Quarnero vor. Die Abgrenzung dieser in der Regel wohl tetraploiden, insgesamt wahrscheinlich heterogenen Art bedarf weiterer Untersuchungen (in der "Flora Iberica" wird - unter dem Namen "Ranunculus ficaria subsp. ficaria " - nicht zwischen F. ambigua und F. grandiflora unterschieden); ob auch die als R. ficaria var. bessarabicus ZAPAE. (von der Dnjestr-Niederung) beschriebenen Pflanzen hierher gehören (PASZKO \& al. 2020) sollte kritisch überprüft werden. Populationen des östlichen Mittelmeergebiets bilden keine Bulbillen (sie wurden als Ranunculus ficaria subsp. chrysocephalus P. D. SELL nach in UK kultivierten Pflanzen beschrieben); aus Italien und der Türkei sind pentaploide Sippen bekannt, aus Georgien wird auch eine hexaploide Chromosomenzahl genannt (PONERT 1975).

\footnotetext{
${ }^{8}$ unter diesem Namen werden aber auch Hybriden (F. ambigua $\times$ grandiflora) gehandelt (vgl. ZONNEVELD 2015).

${ }^{9}$ WAllnÖFER (2008, in Ann. Naturhist. Mus. Wien 109B: 277) hat zwar versäumt, das korrekte Basionym (Ranunculus ficariiformis F.W. SCHULTZ [1855, Arch. F1.: 123 pro syn.] 1858, loc. cit.: 260 ut 'ficariaeformis') zu zitieren; da dieses selbst jedoch auch bei der Veröffentlichung des vermeintlichen Basionyms (R. ficaria subsp. ficariiformis, ebenfalls ein Ersatzname für Ficaria grandiflora; cf. RoUY \& FOUCAUD 1893 Fl. France 1: 73] nicht aufscheint, ist die Kombination nach Art. 41.3 der Nomenklaturregeln (Shenzhen Code, 2018) trotzdem als gültig anzusehen.
} 
Weitere Untersuchungen sind außerdem notwendig, um bessere Einsicht in die Verwandtschaftsverhältnisse zu gewinnen; dabei sind auch die Beziehungen der im Wesentlichen westeuropäischen diploiden $F$. ambigua zur östlich verbreiteten und ebenfalls diploiden $F$. calthifolia abzuklären. Zudem ist das Verhältnis zu F. stepporum P. A. SMIRN. zu klären, die in russischen Floren als weitere diploide Sippe (so etwa bei TZVELEV 2007) behandelt wird.

\section{1b. Ficaria ambigua ${ }^{10}$}

BOREAU 1857, Fl. Centre France, ed. 3, 2: 20. - Syn.: Ranunculus ficaria subsp. fertilis ${ }^{11}$ A.R. ClAPHAM ex LÆGAARD 2001, Nord. J. Bot. 20: 526; Ficaria verna subsp. fertilis (A.R. ClAPHAM ex LÆGAARD) STACE 2009 in Watsonia 27: 246; F. fertilis (A.R. ClAPHAM ex LÆGAARD) GALASSO \& al. 2011 in Atti Soc. Ital. Sci. Nat. 152: 92. - R. ficaria var. fertilis A.R. ClAPHAM 1952 in ClAPHAM \& al., Fl. Brit. Isles: 101, nom. inval. (sine descr. lat.); R. ficaria subsp. fertilis LAWALRÉE 1955 in ROBYNS (Ed.), Fl. Gén. Belgique, Sperm. 2: 59, nom. inval. (sine descr. lat.).

Die Unterschiede zu F. verna s. str. sind dem Bestimmungsschlüssel zu entnehmen. - Chromosomenzahl: $2 \mathrm{n}=16 .-$ Blütezeit: III-IV.

Standort und Vergesellschaftung. Im Vergleich zu $F$. verna (die in England öfters mit gestörten oder anthropogen beeinflussten Standorten in Verbindung gebracht werden kann) ist F. ambigua in alten Laubmischwäldern (in der Regel mit dominierenden Eichen) und in Carpinion-Niederwäldern (in Gesellschaften mit Fraxinus excelsior und Acer campestre) weit verbreitet (TAYLOR \& MARKHAM 1978), außerdem aber auch in frischen Silenion-maritimae-Strandwiesen (öfters in Festuca-rubra-Rasen des Endymio-Armerietum maritimae); auch für Dänemark wird das Vorkommen in Strandwiesen angegeben (LÆGAARD 1966). Gleiches gilt für Südwestfrankreich, wo $F$. ambigua eher an naturnahen Standorten (z. B. in feuchten, halbschattigen Bereichen von Laubmischwäldern) anzutreffen ist, und zwar weit häufiger als $F$. verna (POGAN \& WCISŁO 1975). Nach vorläufigen vergleichenden Kulturversuchen kam NiCHOLSON (1983) zu dem Schluss, dass $F$. ambigua lichttoleranter ist als $F$. verna; letztere beginnt an sonnexponierten Standorten früher zu blühen, aber auch (bei spärlicher Bulbillen-Entwicklung) vorzeitig einzuziehen, während $F$. ambigua an lichtoffenen gegenüber Schatt-Standorten nur unwesentliche Unterschiede bezüglich der Fruchtentwicklung zeigt.

Allgemeine Verbreitung. Atlantisches Europa, von den Britischen Inseln, dem westlichen Belgien und Frankreich bis zur Iberischen Halbinsel; außerdem sind einzelne Vorkommen in Südwest-Norwegen und Dänemark bekannt (nicht in den Niederlanden: vgl. ANDREAS 1966!); vermutlich auch in Nordwestafrika. Für fraglich hält SELL (1994) die Angaben in der »Med-Checklist« (GREUTER \& al. 1989) und im 8. Heft des »Atlas Florae Europaeae «(JALAS \& SUOMINEN 1989: 185; Rasterkarte) für Korsika, Sardinien und Sizilien sowie die Apenninen-Halbinsel, obwohl für Korsika ein karyologischer Nachweis (POGAN \& WCISŁO 1986) vorliegt.

Verbreitung im Gebiet. Sichere Vorkommen sind im grenznäheren Gebiet nur aus Dänemark (nordwestl. Jütland, so v. a. um Limfjorden) bekannt.

\footnotetext{
10 lat. ambiguus »zweideutig«.

11 lat. ferrtilis »fruchtbar«; im Gegensatz zu F. verna, die fast nie keimfähige Nüsschen ausbildet.
} 
Kritisch zu überprüfen ist die Identität der ursprünglich unter dem Namen „F. ranunculoides var. incumbens" bekannt gemachten Vorkommen eines fruchtenden, bulbillenlosen Scharbockskrauts um Wissembourg [Weißenburg] (SCHULTZ 1855: 122, der seine Varietät allerdings mit $F$. calthifolia gleichsetzte; vgl. auch VOLLMANN 1914: 275). Dieses Vorkommen im Unterelsass hat aber seither offenbar keine nähere Beachtung mehr gefunden.

Ob die einzige bisher aus der Schweiz (Justistal nordöstlich von Merligen am Thuner See) nachgewiesene diploide Population von „Ranunculus ficaria agg.“ möglicherweise hierher zu ziehen ist, muss offen bleiben, da die Autorin (TRÖHLER 1976) keine weiteren Angaben machte und neuere Untersuchungen fehlen.

Lebensgeschichte. Auf den Britischen Inseln beginnt die aktive Vegetationszeit mit der Laubentfaltung Mitte Januar, nachdem die Erneuerungsknospen bereits im Dezember ergrünen und sich zunehmend vergrößern. Die Hauptblütezeit ist um die Wende März/April (TAYLOR \& MARKHAM 1978). In den gleichen Zeitraum fällt die Keimung vorjähriger Diasporen (MARSDEN-JONES 1935); sie ist von den Lichtverhältnissen unabhängig, wird jedoch durch eine Kälteperiode begünstigt. Die Nüsschen keimen gut (Keimungsrate im Experiment meist $>70$ \%). TAYLOR \& MARKHAM beobachteten im Juni zahlreiche „Jährlinge“ um entsprechende Mutterpflanzen. Jungpflanzen blühen frühestens im 2. Jahr, in der Mehrzahl aber erst im 3. Jahr. Phänologisch verhalten sich in England $F$. ambigua und $F$. verna gleich. Im Frühsommer (mit höheren Temperaturen, zunehmender Tageslänge und Strahlungsintensität) beginnen die Pflanzen einzuziehen.

\section{Ficaria calthifolia ${ }^{12}$}

REICHENBACH 1832, Fl. Germ. Excurs.: 718 [ut 'calthaefolia'] - Syn.: Ranunculus calthifolius (RCHB.) BlufF \& al. 1837 in BlufF \& FInGERHUTH, Comp. Fl. Germ. ed. 2, 1(2): 295; R. ficaria var. calthifolius (RCHB.) GuSS. 1844, Fl. Sic. Syn. 2: 41 [ut 'calthaefolius']; R. ficaria var. peltiformis NEILR. 1859, Fl. Nieder-Oesterr.: 685, nom. illeg. (superfl.); Ficaria nudicaulis A. KERN. 1863, Oesterr. Bot. Z. 13: 188; F. intermedia SCHUR 1866, Enum. Pl. Transsilv.: 14; F. transsilvanica SCHUR 1866, op. cit.: 14; F. verna subsp. calthifolia (RCHB.) NYMAN 1878, Consp. Fl. Eur.: 7 [ut 'calthaefolia']; Ranunculus ficaria subsp. calthifolius (RCHB.) ARCANG. 1882, Comp. Fl. Ital.: 11 [ut 'calthaefolius']; Ficaria pumila VelEn. 1887, Sitzungsber. Königl. Böhm. Ges. Wiss. Prag, Math.-Naturwiss. Cl. 1887: 438; F. verna subsp. pumila (VELEN.) VELEN. 1891, Fl. Bulg. 1: 6; Ranunculus ficaria subsp. nudicaulis (A. KERN.) ROUY \& FOUCAUD 1893, Fl. France 1: 73; Ficaria ledebourii GROSSH. \& SCHISCHK. 1930 in GROSSHEIM, Fl. Kavkaza 2: 108; F. verna subsp. ledebourii (GROSSH. \& SCHISCHK.) SoÓ 1966, Acta Bot. Acad. Sci. Hung. 12: 111, comb. inval. (sine relat. basion.). - Nacktstängliges Scharbockskraut. - Ital.: ficaria a fusto nudo; slowen.: golostebelna lopatica; ungar.: salátaboglárka; slowak.: blyskáč záružl'olistý; tschech.: orsej blatoucholistý; poln.: ziarnopłon kusy; russ.: чистяк калужницелистный [čistjak kalužnicelistnyj]. - Abb.: $\rightarrow$ KÄSTNER \& FISCHER 2006, Abb. 4, www.zobodat.at/pdf/NEIL 4 0075-0109.pdf, $\rightarrow$ DRENCKHAHN 2016, Abb. 1, 12, http://www.forumgeobotanicum.net/articles/vol 7-2016/drenckhahn ficaria/drenckhahn ficaria calthifolia.html.

Pflanze ausdauernd (Wurzelknollen-Geophyt), Wuchshöhe (5-)7-15(-20) cm. Wurzelstock (Grundachse) extrem verkürzt, mit einem dichten Bündel zahlreicher \pm birn- bis keulenförmiger, meist $1-4(-7) \mathrm{cm}$

12 lat. -folius »-blättrig«; die Form der Laubblätter erinnern an jene von Caltha palustris. 
langer, bis etwa $7 \mathrm{~mm}$ dicker Speicherwurzeln und einem Kranz jährlich neugebildeter, dünner $(<1 \mathrm{~mm})$, verzweigter Faserwurzeln. Jahresspross frühjahrsgrün, Achse manchmal fast völlig gestaucht oder mit einem kurzen, meist 2-4(-7) cm langen, kräftigen (oft 3-5 mm $\varnothing$ ) subterranen Stängelinternodium; oft einzeln oder aber mehrere pro Wurzelbündel. Grundständige Blätter wenige: meist 2-3(-6) schmal dreieckig-lanzettliche, 1-2(-4) cm lange, scheidenartige, häutige Niederblätter und 1-2 lang gestielte, am Grund scheidig verbreiterte Laubblätter; die meist zahlreichen (2-6 oder mehr) weiteren, ebenfalls lang (meist 5-12[-16] cm) gestielten Laubblätter (einschließlich der Vorblätter der Seitensprosse) an der Basis des Sprosses rosettig gedrängt, an der Bodenoberfläche oder knapp darunter entspringend; Brutknollen (Bulbillen) in den Blattachseln stets fehlend. Blattspreiten breit eiförmig (bis eiförmig-rundlich), vorn stumpf gerundet, mit herzförmigem Grund und häufig enger (manchmal überlappter) Bucht, etwas länger als breit (größte Spreiten ca. 3-41/2 $\times 2 \frac{1}{2}-4 \mathrm{~cm}$ ); Blattrand sehr seicht und entfernt gekerbt, gegen den Grund zu gelegentlich schwach lappig gekerbt. Blüten (eines Jahressprosses) (1-)3-5(-6), meist 6-12 cm gestielt, Blütenstiele i. d. Regel direkt der Blattrosette entspringend (selten mit einem kurzen Stängelglied und deutlich reduziertem Stängelblatt), zur Fruchtzeit bogig zum Boden hinabgekrümmt. Äußere Blütenhüllblätter (Kelchblätter) 3(-5), breit eiförmig, (5-)7-8(-10) × 4-6(-7) mm, aus häutigem Grund krautig und in den vorderen Dritteln grün. Innere Blütenhüllblätter (Kronblätter) (7-)8-11(-17), elliptisch (-eiförmig), (9-)11-15(-18) × 4-6(-8) mm; Nektargruben 1,0-1,2 mm lang. Staubblätter meist 25-30 (-50). Fruchtblätter (10-)15-25(-35), i. d. Regel alle sich wohl entwickelnd; reife Nüsschen (einschl. der stielartig verschmälerten Basis) $2 \frac{1}{2}-3 \frac{1}{2} \mathrm{~mm}$ lang und $2-2 \frac{1}{2} \mathrm{~mm}$ dick, kugelig gewölbt, nur schwach gekielt, \pm locker kurz behaart, Griffelspitze undeutlich (bis 0,2 mm). - Chromosomenzahl: 2n $=16^{13}$. - Blütezeit: (II-) III-IV (Beginn der Anthese ca. 2 Wochen früher als $F$. verna s. str.).

Standort und Vergesellschaftung. In Mitteleuropa in der [planar-]collinen Stufe (in Mähren bis 360 m, in der grenznahen Slowakei bis $550 \mathrm{~m}$ ); einzeln oder gruppenweise als mäßig heliophile Pflanze vor allem im Randbereich oder in Saumgesellschaften thermophiler Eichen-Hainbuchen- und anderer lichter Eichenmischwälder (v. a. Flaumeichen-Buschwald), seltener auch in lichten Aue-Gebüschen (Hartholzau im Übergang zu Eichenmischwäldern); aus den Gebüsch-Gesellschaften auch in tiefgründige Wiesensteppen (etwa über Löss; auch über Salzböden) und Halbtrockenrasen vordringend, so schließlich auch (teilweise wohl als „Relikt“ ehemaliger Gebüsche und Hecken) in Parkrasen und anderen anthropogenen Wiesen. Dem entsprechen in der »Flóra Slovenska« angeführte phytosoziologische Zuordnungen (FUTÁK 1982): Carpinion betuli und Quercion pubescenti-petraeae sowie Alnion incanae [,Alno-Padion“] als Gehölz-Verbände, Festucion pseudovinae und Alysso saxatilis-Festucion pallentis [„Asplenio-Festucion glaucae“] als Rasen-Verbände; in der »Květena Česke SR « wird auch Arrhenatherion [einschl. „Alopecurion pratensis“] genannt (KŘísA 1988).

Allgemeine Verbreitung. Südöstliches Mitteleuropa: östliches Österreich, Süd-Mähren und angrenzende Slowakei (zerstreut vom Marchfeld durch die Süd-Slowakei; Punktkarte: FUTÁK 1982: 218) sowie Ungarn; isolierte Teilareale nördlich davon in Böhmen, in Deutschland (mittleres Elbtal, vgl. unten!) und Polen (außer in Schlesien noch auf der Kleinpolnischen Höhe zwischen Krakau und Kielce sowie bei Przemyśl; Punktkarte: TOWPASZ 1971: 217, Rasterkarte: ZAJĄC \& ZAJĄC 2001: 240); durch Südosteuropa (Serbien, Rumänien, Bulgarien) bis zur Krim und Kolchis; in Oberitalien im östlichen Alpenvorland (vom Gardasee? ${ }^{14}$ bis Friaul), im angrenzenden Slowenien, auf der Balkanhalbinsel von

\footnotetext{
13 Die Angabe, dass $F$. calthifolia in Ungarn 2 n = 32 besäße (SoÓ \& BORHIDI 1966), wurde nie bestätigt. Vgl. aber im Folgenden unter ,Variabilität“!

14 ein allerdings noch zu verifizierender Neufund bei Sirmione (EMILIO ZANOTTI, Brescia, in litt.).
} 
Triest und Istrien über Dalmatien bis in die Gebirge Nord-Griechenlands (auch auf den westgriechischen Inseln), westlich der Adria im Apennin (sicher zumindest im Umbrischen Apennin, in den Abruzzen und südlich bis zur Sila); angeblich auch in Sizilien und Korsika. (Angaben von der Iberischen Halbinsel - aus der Estremadura: VÁSQUEZ PARDO 2016 - bedürfen einer kritischen Überprüfung.) In Nordamerika bereits im 19. Jahrhundert eingeschleppt (ältester Beleg - aus Pennsylvanien - von 1867; stellt wie $F$. verna s. str. je ein Drittel der aus den USA bekannten Belege des $F$. verna-Komplexes; vgl. PoST \& al. 2009).

Zonale Arealdiagnose: sm-(temp)·oz(2-)3 EUR

Regionale Arealdiagnose: (zentral-)ostsubmed-(pont-südostsarm?)-pann-bohem-südpolon

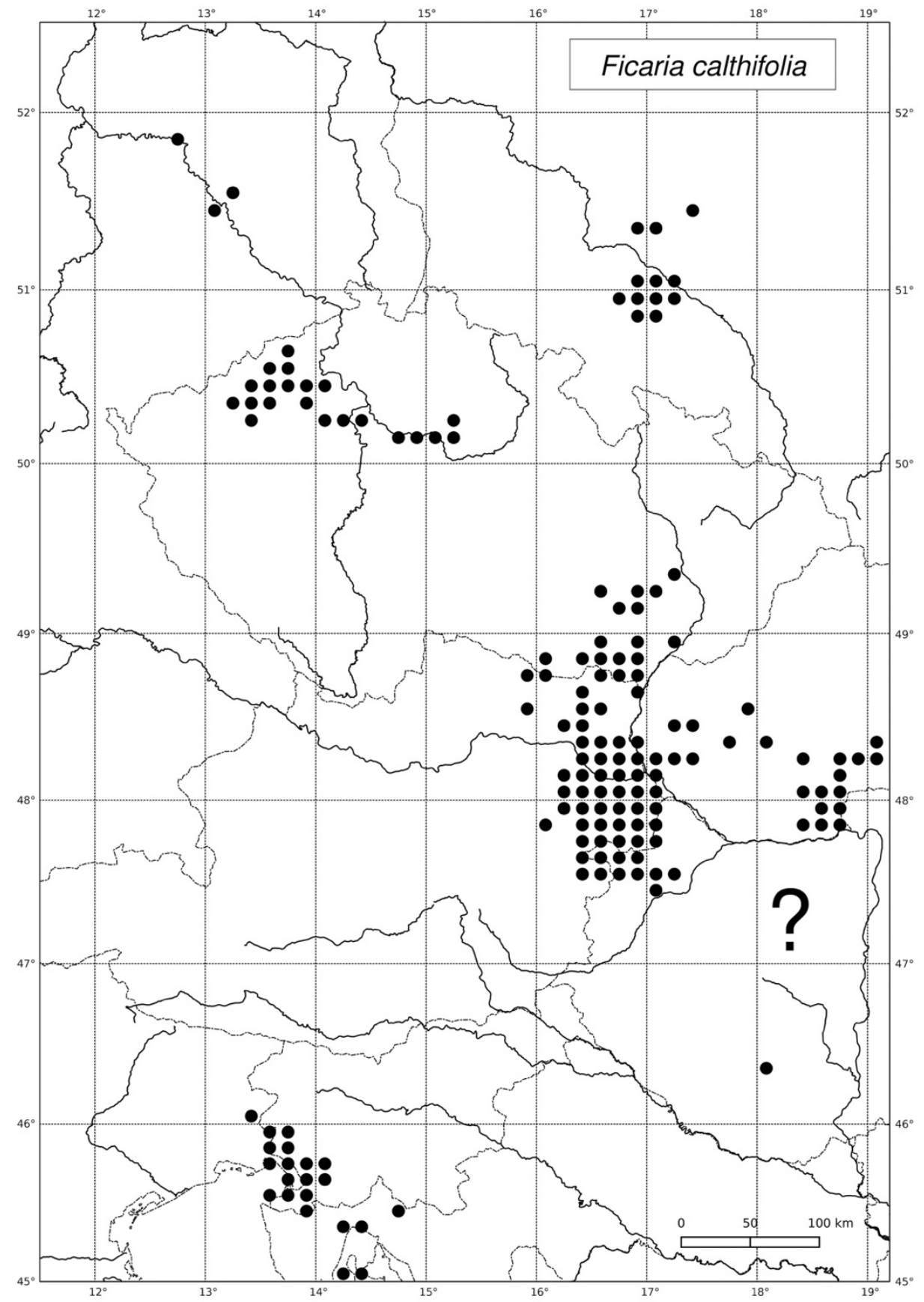

Abb. $2 \mid$ Verbreitung von Ficaria calthifolia in Mitteleuropa (Rasternetz der Kartierung der Flora Mitteleuropas, Vorkommen bezogen auf „Grundfelder“ von 10 ‘ geogr. Länge $x$ $6^{\prime}$ geogr. Breite) 
Verbreitung im Gebiet. In Österreich nur im pannonisch geprägten östlichen Niederösterreich (Weinviertel nordwestlich bis Retz, Wiener Becken mit Randlagen) und nördlichen bis mittleren Burgenland (südlich bis um Großwarasdorf); anschließend an die Vorkommen im Weinviertel in Tschechien (Rasterkarte: SLAVÍK 1986: 87) im pannonischen Süd-Mähren, nördlich davon nur im Mittelböhmischen Becken sowie in Polen in Niederschlesien (um Breslau); in Oberitalien im südöstlichen Friaul und um Triest (Rasterkarte: POLDINI 2002: 401) (nach PIGNATTI 1982 westlich davon auch im Veneto) sowie im angrenzenden Teil Sloweniens (Rasterkarte: JoGAN 2001: 310). Für die neuerdings bekannt gewordenen Vorkommen in Deutschland im Elbtal bei Nünchritz und bei Mühlberg (ILLIG \& RisTOW 2015, DRENCKHAHN 2016) sowie flussabwärts bei Pretzsch (FRANK 2017) wird Verschwemmung durch historische Hochwässer diskutiert; für jenes in Würzburg kann eine anthropogene Verschleppung angenommen werden (DRENCKHAHN 2016). Zur Verbreitung in Mitteleuropa s. $\rightarrow$ Abb. 2.

Lebensgeschichte. Die Laubentfaltung beginnt bei der meist in Gruppen (aber nicht in deckenden Herden) auftretenden $F$. calthifolia bereits im Vorfrühling, gewöhnlich schon im Februar. Rasch entwickeln sich auch die bereits im Herbst angelegten Blütenknospen: der Beginn der Anthese liegt ca. 2 Wochen früher als bei $F$. verna; im Mai sind die Pflanzen in der Regel bereits \pm völlig eingezogen. Die Wuchsrhythmik entspricht prinzipiell jener von $F$. verna. Nach der Winterpause verläuft die Pollen- und Embryosackentwicklung ungestört, desgleichen setzt nach Fremdbestäubung die Samenentwicklung rasch ein (POGAN \& WCiSŁO 1981a, WCISŁO \& POGAN 1981). Die Karpelle entwickeln sich in hohem Maß zu wohlausgebildeten Nüsschen. Die oft schon vor der vollständigen Samenreife zum Substrat hinabgekrümmten Fruchtstiele „versenken“ die Sammelfrucht häufig auf den Boden und entsprechen so einem myrmekochoren Syndrom. Die Keimung der Nüsschen setzt nach der Sommerruhe ein; ein Aussaatversuch WOLTERs (1933) Anfang Juni zeigte erste Sämlinge Ende Oktober, 23 Wochen nach dem Saattermin waren $93 \%$ der Nüsschen gekeimt. Von lufttrocken gelagerten Nüsschen waren im 3. Jahr nur noch $14 \%$ keimfähig. Die Beobachtungen von WINKLER $(1926,1927)$ anhand seiner Experimente mit mehreren Tausend um Breslau gesammelter „reifer Scharbocksfrüchte“ dürften sich, vermutlich ebenso wie jene oben zitierten von WOLTER (1933), zumindest auch (wenn nicht zumeist) auf die dort vorkommende $F$. calthifolia beziehen. Im Kalthaus erschienen erste Keimblätter seiner Aussaat bereits im Dezember, im Januar waren über 50 \% der im vorjährigen Mai gereiften Früchte zu Keimpflanzen entfaltet. Die Sämlinge der Freilandpflanzen erschienen im Frühjahr und hatten vor der neuerlichen Sommerruhe die erste Wurzelknolle gebildet (nur ausnahmsweise schon ein Laubblatt); die eigentliche Laubentwicklung (mit Nieder- und Laubblättern) und weitere Knollenbildung setzte mit der 2. Vegetationsperiode ein. Die Jungpflanzen kommen bei entsprechender Erstarkung wohl frühestens im Folgejahr erstmals zur Blüte.

Taxonomie. Angesichts der „deutlichen morphologischen Unterschiede, [der] verschiedenen Fortpflanzungsbiologie und ... ausgeprägten ökogeographischen Unterschiede" sind wir mit KÄSTNER \& FISCHER (2006) der Meinung, dass der Sippe gegenüber F. verna Artrang gebührt. Triploide Hybriden mit F. verna s. str. treten zwar an Kontaktstellen auf, doch kommt es allem Anschein nach nicht zu einer Verwischung der Diskontinuität, weil die Hybriden weitestgehend (in noch höherem Maß als die Tetraploiden selbst) steril sind (POGAN \& WCISŁO 1983; POPELKA \& al. 2019). Dementsprechend gab es schon früher, gerade in Gebieten sympatrischen Vorkommens, die Tradition, beide Sippen als Arten zu behandeln (KERNER 1863 sowie 1887 in seinen »Schedae ... « [no. 1728-1729] oder FRITSCHs »Excursionsflora für Oesterreich« 1897, auch in der 3. Auflage: 1922; PACZOSKI 1927 in der »Flora Polska« SZAFERs). Bezüglich der Selbständigkeit dieser Ficaria-Arten sei als historisch interessante Notiz erwähnt, dass bereits GREGOR MENDEL im Rahmen seiner Untersuchungen zum „Lamarckismus“ mit den 
beiden in Mähren heimischen Sippen (also F. verna und F. calthifolia) experimentiert und deren von Umwelteinflüssen unabhängige Konstanz über Jahre hinweg beobachtet hatte.

Nomenklatur. Der auf dalmatinische Pflanzen gegründete Name ist noch nicht typisiert, wird jedoch allgemein in dem hier definierten Sinn angewandt und orientiert sich an der später (REICHENBACH 1838/39: 9, t. I) in den »Icones ... « publizierten Abbildung (no. 4571).

Variabilität. F. calthifolia variiert in Mitteleuropa weniger auffällig als $F$. verna, doch sind auch hier in der Ausbildung der Spreite (besonders des Blattrands: nahezu ganzrandig versus lappig gekerbt) Unterschiede zwischen einzelnen Populationen zu konstatieren, ohne dass dem taxonomische Bedeutung beizumessen wäre. Gleiches gilt für die Blütengröße (Länge und Breite der Kronblätter); entsprechende Abweichungen von der „Norm“ lassen es auch nicht zu, den Gesamteindruck einer größeren, ,,volleren“ Blüte (mit einander \pm überdeckenden Kronblättern) von $F$. calthifolia gegenüber $F$. verna als diakritisches Merkmal zu verwenden. DRENKHAHN (2016) unterscheidet bei den in Deutschland etablierten Vorkommen außer typischer (diploider) F. calthifolia („Typ1“) durch robusteren Wuchs, größere Blüten und reduzierter Fruchtbarkeit abweichende Populationen: diese sind entweder triploid („Typ2“) oder (vermutlich) pentaploid („Typ3“). Gleichermaßen von diploider F. calthifolia abweichende Zytotypen, nämlich triploide und tetraploide Pflanzen, wurden von den Ionischen Inseln bekannt (DRENCKHAHN \& al. 2017).

\section{Danksagung}

Der Autor dankt M. A. FischeR, H. GReger, J. Greilhuber, H. HALbRitTeR, H. NiKLFeld, H. VOGLMAYR, A. WEBER (alle: Fakultätszentrum für Biodiversität, Wien) und H. TEPPNER (Institut für Pflanzenwissenschaften, Graz) für einschlägige Hinweise und Ergänzungen, Hilfe bei der Literaturbeschaffung und kritische Durchsicht der früheren Fassungen des Manuskripts $(2008,2010)$. D. DRENCKHAHN (Würzburg) bin ich für anregende Diskussion und einschlägige Hinweise verpflichtet. Gleiches gilt für die Herausgeber der vorliegenden Fassung, J. W. KADEREIT (Mainz) und insbesondere E. J. JÄGER (Halle), der entsprechende Versionen über die Jahre kritisch begleitet und die chorologische Diagnostik hilfreich ergänzt hatte. M. HOFBAUER (Wien) half sachkundig bei der Erstellung der Verbreitungskarte; Frau D. FRANKE (Mainz) besorgte umsichtig die Formatierung der Letztfassung. 